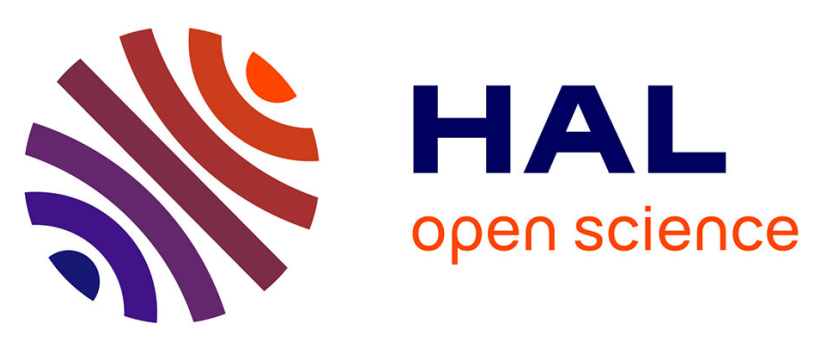

\title{
Visco-acoustic modelling of a vibrating plate interacting with water confined in a domain of micrometric size
}

Bérengère Lebental, Frédéric Bourquin

\section{To cite this version:}

Bérengère Lebental, Frédéric Bourquin. Visco-acoustic modelling of a vibrating plate interacting with water confined in a domain of micrometric size. Journal of Sound and Vibration, 2012, 331 (8), pp 1870-1886. 10.1016/j.jsv.2011.12.016 . hal-00854222

\section{HAL Id: hal-00854222 \\ https://hal.science/hal-00854222}

Submitted on 26 Aug 2013

HAL is a multi-disciplinary open access archive for the deposit and dissemination of scientific research documents, whether they are published or not. The documents may come from teaching and research institutions in France or abroad, or from public or private research centers.
L'archive ouverte pluridisciplinaire HAL, est destinée au dépôt et à la diffusion de documents scientifiques de niveau recherche, publiés ou non, émanant des établissements d'enseignement et de recherche français ou étrangers, des laboratoires publics ou privés. 


\title{
Visco-acoustic modelling of a vibrating plate interacting with water confined in a domain of micrometric size
}

\author{
B. Lebental ${ }^{*, *, a, b, c}$, F. Bourquin ${ }^{\mathrm{a}}$ \\ ${ }^{a}$ Université Paris-Est,IFSTTAR, 58 Bld. Lefebvre, 75732 Paris Cedex 15, France \\ ${ }^{b}$ CEA-LETI, MINATEC, 17 rue des Martyrs, 38054 Grenoble, Cedex 9, France \\ ${ }^{c}$ ENPC-ParisTech, Av. Blaise Pascal, Cité Descartes, 77455 Marne-la-Vallée Cedex 2, France
}

\begin{abstract}
It is well established that concrete durability strongly depends on the capillary porosity of the material. Hence, structural health monitoring of concrete structure could take advantage of concrete microporosity monitoring. To this end, a new method for the in situ non-destructive testing of capillary porosity in cementitious materials has been proposed. A sensing device that seems well suited to this application is a capacitive ultrasonic transducer with a characteristic size of $1 \mu \mathrm{m}$. It is to be embedded in the material. Its vibrating membrane is made of aligned carbon nanotubes forming a thin layer with a typical thickness of $1 \mathrm{~nm}$. It generates acoustic waves of micrometric wavelength into water-filled micropores, aiming at measuring their properties.
\end{abstract}

The present paper focuses on the numerical simulation of the embedded sensor. In order to properly account for viscous effects in fluids at the micrometric scale, we have developed a specific computational method for the visco-acoustic modelling of a microplate vibrating between $10 \mathrm{MHz}$ and $2 \mathrm{GHz}$ in a water-filled domain of micrometric size. Our approach is based on the condensation of the fluid part of the fluid-structure problem on the structure by a finite element method, and on a spectral approximation of the structural equations.

The numerical results indicate that the fluid domain is resonant despite the viscous terms, which causes a frequency downshift of the resonances and a decrease of the quality factor. In the coupled system, the plate does not perturb the fluid resonances, whereas the plate resonances are strongly upshifted by the water load. The resonance frequencies of the system are shown to display a clear dependence on

\footnotetext{
${ }^{*}$ Corresponding author

** The authors thank J. Fang for his contribution on the implementation of the 3D method.

Email addresses: berengere. lebentaleifsttar.fr (B. Lebental), frederic.bourquin@ifsttar.fr (F. Bourquin)
} 
the pore width, which makes the device a good candidate as a porosity sensor.

Key words: fluid-structure interaction, elasto-acoustics, microfluidics, ultrasonic transducer, viscosity, water

PACS: 02.60.Cb, 43.35.+d, 43.38.+n, 47.61.-k

\section{Introduction}

Within the framework of structural health monitoring for civil engineering [1, 2], a new method $[3,4]$ for the non-destructive testing of capillary porosity of cementitious materials has been proposed. It is based on dispersing in the studied material a large number of microsystems equipped with specific sensing devices able to collect microscale information on the material microstructure.

One of the sensing devices envisioned for this application is a high frequency capacitive Micromachined Ultrasonic Ttransducer (cMUT) with a characteristic size of $1 \mu \mathrm{m}$. Its vibrating membrane is made up of aligned carbon nanotubes $[4,5]$; it has a high Young's modulus $(0,5$ to $1 \mathrm{TPa})$ and a low thickness ( 1 to $5 \mathrm{~nm}$ ). The waves emitted by this prospective device are expected to propagate mostly in the fluid component of the microstructure. Due to its micrometric size, it should be able to excite pores individually, so that the collected information should be resolved at the microscale. From the measurements provided by a large number of such transducers, one plans to derive statistically morphological information on the microporosity [6].

The present paper describes a numerical visco-acoustic model suited for the design of such a device and its use in a confined fluid medium of micrometric size. More precisely, the method yields the shape and the amplitude of membrane vibrations depending on the medium and the membrane characteristics. The ultimate purpose of this method, which lies beyond the scope of this paper, is to optimize technological parameters and to identify potentially observable characteristics of the porosity.

First, we identify the relevant equations from the literature. We then suggest a formulation for the elasto-acoustic problem and a fast numerical method, whose convergence is studied numerically. Finally, we present the results obtained by the method.

Main result is the resonant behaviour of the fluid system, which is only weakly perturbed by coupling with the plate. The plate behaviour is strongly perturbed by the presence of the fluid, which induces a significant added stiffness effect on the plate. 
The resonance frequencies of the system are shown to display a clear dependence on the pore width, which makes the device a good candidate as a porosity sensor.

\section{State of the art}

\subsection{Non viscous modelling of cMUT devices}

A cMUT device consists of a metallic microplate suspended above an electrode and actuated by capacitive effect. When one considers the behaviour of a cMUT device interacting with a fluid environment, the fluid medium is most often modelled in the framework of non-viscous wave acoustics: the dissipative terms of Navier-Stokes equations are fully neglected, so that the fluid system is ruled by the d'Alembert wave equation with Dirichlet boundary conditions for the normal velocity only.

\subsection{Significant role of the dissipative terms in microfluidics}

The scale of the fluid domains (100 $\mathrm{nm}$ to $10 \mu \mathrm{m})$ considered in the present study anchors us in the field of microfluidics: the Reynolds number, proportional to the characteristic size of the fluid domain, is much smaller than at the macroscopic scale. Hence one expects that dissipative terms, negligible in larger systems, cannot be neglected in microfluidics.

This is confirmed experimentally: water flows in micro-channels with a size ranging between $2 \mu \mathrm{m}$ and $100 \mu \mathrm{m}$ display a boundary layer with thickness comparable with the size of the channel[7, 8]. The acoustic behaviour of fluids in the microchannels of ink-jet printers (with diameter typically ranging from $10 \mu \mathrm{m}$ to $100 \mu \mathrm{m}$ ) is in good agreement with a narrow-channel model including fluid viscosity [9].

\subsection{Visco-thermal elasto-acoustics}

These elements point out the need to integrate viscous terms in the fluid equations: it is the purpose of visco-thermal acoustics, which is based on the use of the fully-linearized Navier Stokes equations $[10,11,12,13,14,15]$. From the fully linearized equations, several simplified models can be derived [12], such as the narrow-channel [9], the boundary layer impedance [16], the low-reduced-frequency [17] or the low-Reynold's number [13] models.

The solutions to the visco-thermal acoustic equations can be determined analytically for specific geometries [11] and for some of the simplified models. Several numerical methods based on finite elements $[14,15]$ and boundary elements $[18,19]$ are also available. 
These methods have been extended to elasto-acoustics: for simple 2D or axisymmetrical 3D geometries and for simplified visco-thermal acoustic models, analytical solutions to the elasto-acoustic problem may be derived $[20,12,13]$. The fully-linearized elasto-acoustic problem is usually solved numerically with boundary elements [21]. For some simplified problems, such as the boundary layer impedance [16] and the narrow channel models [20], finite elements based methods have also been developed.

The specificity of our elasto-acoustic problem (higher frequencies, larger bandwidth and lower size, water instead of air) excludes the use of any of the available simplified models. Moreover, there is no proof that the rotational velocity can actually be neglected over the whole range of parameters, which rules out the use of the existing boundary elements methods.

These remarks justify the introduction of a different approach for visco-thermal elasto-acoustic numerical modelling, which will be discussed in the rest of this paper. The main originality of our approach is to solve the fully-linearized visco-thermal elasto-acoustic problem using a mixed finite elementspectral approach, which do not require the assumption of null rotational velocity and is significantly more flexible than boundary elements.

\subsection{Breakdown of continuity in water microfluidics}

In order for the Navier Stokes equations to describe properly a fluid, the fluid must behave as continuous medium. The Knudsen number $K_{n}=\frac{\text { mean free path }}{\text { characteristic size }}$ indicates whether a continuous model is adapted $\left(K_{n}<0.01\right)$ or if a semi-discrete $[22,23]$ or discrete $[24,25,26]$ model is needed to account for nanoscale phenomena such as adsorption on walls, electrostatic forces or steric exclusion [27, 28, 29].

In the case of liquid water at ambient pressure and temperature, the mean free path is around $2 \AA$, so that the continuity hypothesis theoretically stands down to $200 \mathrm{~nm}$ of characteristic size. A metaanalysis of published experimental data has validated this hypothesis [30]. As a consequence, as we consider only a system with characteristic size larger than $100 \mathrm{~nm}$, we do not take into account in our problem the possibility of continuity breakdown.

\section{Formulation of the elasto-acoustic problem}

Notations: if $f$ is a given function on the domain $\Omega$, let $<f>=\frac{1}{\operatorname{Vol}(\Omega)} \int_{\Omega} f(M)$ denote its average on $\Omega, \delta f=f-<f>$ the variations around the average, $\tilde{f}$ a non-dimensional function associated to $\delta f$ 
and $F$ the Fourier transform of $\tilde{f}$. If $f$ is a complex number, let $\bar{f}$ be its conjugated complex and $|f|$ its modulus. Vectors, gradient, tensors and matrices are written in bold letters. Let us write $i=\sqrt{-1}$.

\subsection{Geometry}

We consider a clamped plate with one free side. Its other side is in contact with distilled water filling a closed domain with straight boundaries. The closed domain stands for a smooth-walled, unconnected pore of the studied material surrounded by an infinitely rigid matrix.

The plate is rectangular with size $l_{x}$ and $l_{y}$. The fluid domain (pore) $\Omega$ is a rectangular parallelepiped with size $l_{x}, l_{y}$ and $l_{z}$ (Figure 1 left). The plate forms the left side of the parallelepiped, denoted $\Gamma^{s}$. Let us denote $\partial \Omega$ the boundary of $\Omega, \Gamma^{f}=\partial \Omega-\Gamma^{s}$ and $\partial \Gamma^{s}$ the boundary of $\Gamma^{s}$. In the rest, we call $l_{x}$ the domain height, $l_{y}$ its depth and $l_{z}$ its width.

For the sake of computational efficiency, we resort to twodimensional computations for the parametric studies. In $2 \mathrm{D}$, the chosen model geometry is a section of the $3 \mathrm{D}$ domain (Figure $1 \mathrm{right}$ ), that is a rectangle with height $l_{x}$ and width $l_{z}$.
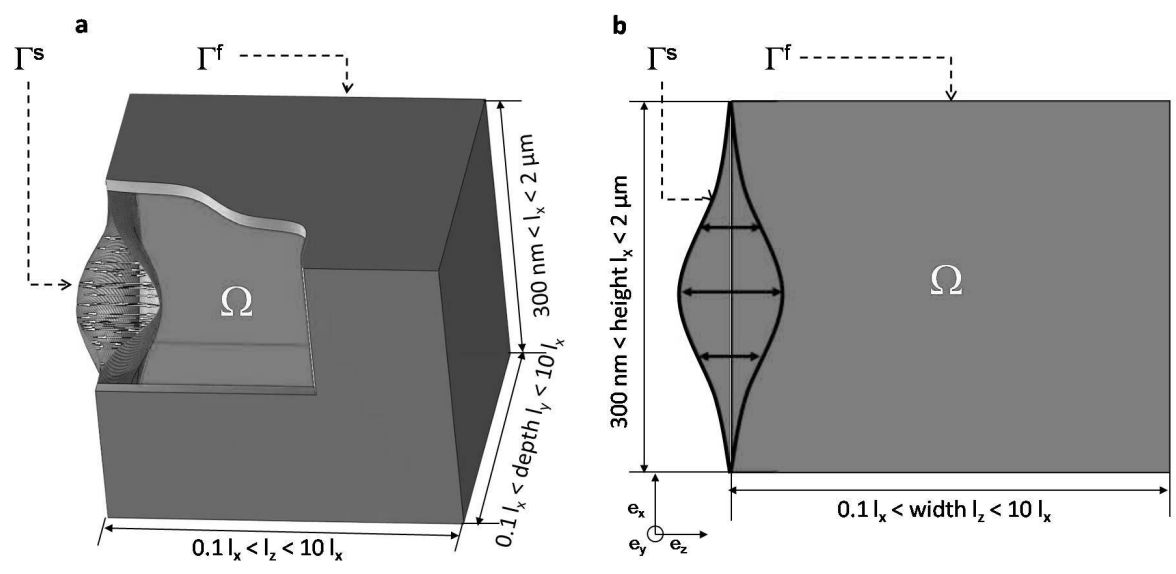

Figure 1: Model geometries: (a) 3D setting; (b) 2D setting.

\subsection{Fluid equations}

\subsubsection{Notations}

Let us first establish the equation that govern the fluid evolution. We assume that the water in the pore behaves like a compressible Newtonian fluid. There is no mean flow nor volume forces nor heat generation. Acoustic flows are laminar. 
The state variables of the fluid are $p$ the pressure, $\rho^{f}$ the volume mass, $\theta$ the temperature, $\boldsymbol{u}^{f}$ the velocity. Let us denote $\delta p, \delta \rho^{f}, \delta \theta$ and $\delta u^{f}$ the small amplitude variations of the state variables around their average values $p_{0}, \rho_{0}^{f}, \theta_{0}$ and $\boldsymbol{u}_{0}^{f}=\mathbf{0}$. Depending on the context, let us call grad the 2D or 3D gradient and Id the $3 \times 3$ or $2 \times 2$ identity tensor. According to these definitions, the tensors $\boldsymbol{e}\left(\boldsymbol{\delta} \boldsymbol{u}^{f}\right)=$ $\frac{1}{2}\left(\operatorname{grad} \delta \boldsymbol{u}^{f}+\operatorname{grad}^{t} \delta \boldsymbol{u}^{f}\right)$ and $\delta \boldsymbol{\sigma}=\left(-\delta p+\lambda \operatorname{div} \delta \boldsymbol{u}^{f}\right) \boldsymbol{I} \boldsymbol{d}+2 \mu \boldsymbol{e}\left(\boldsymbol{\delta} \boldsymbol{u}^{f}\right)$ denote respectively the small amplitude variations of the linearized strain and stress tensors.

The system is studied at ambient pressure $p_{0}=1$ atm and ambient temperature $\theta_{0}=20^{\circ} \mathrm{C}$. The following parameters are assumed to be constant over $\Omega$ : density $\rho_{0}^{f}=1000\left(\mathrm{~kg} \cdot \mathrm{m}^{-3}\right)$, speed of sound $c=1500 \mathrm{~m} . \mathrm{s}^{-1}$, dynamic viscosity $\mu=1.0 \times 10^{-3} \mathrm{~kg} \cdot \mathrm{m}^{-1} \cdot \mathrm{s}^{-1}$, second viscosity $\lambda=\frac{-2}{3} \mu=-6.7 \times$ $10^{-4} \mathrm{~kg} \cdot \mathrm{m}^{-1} \cdot \mathrm{s}^{-1}$, thermal conductivity $K=0.62 \mathrm{~W} \cdot \mathrm{m}^{-1} \cdot \mathrm{K}^{-1}$, isothermal compressibility $\kappa_{T}=4.6 \times$ $10^{-10} \mathrm{~Pa}^{-1}$, isobaric specific heat $C_{p}=4.2 \times 10^{3} \mathrm{~J} \cdot \mathrm{kg}^{-1} \cdot \mathrm{K}^{-1}$, isobaric thermal expansion coefficient $\alpha=2.1 \times 10^{-4} \mathrm{~K}^{-1}$.

\subsubsection{Linearized equations}

According to widespread literature on visco-thermal acoustics (for example [11, 12]), the fluid behaviour can be described by the following equations:

$$
\begin{array}{r}
\partial_{t} \delta \rho^{f}+\rho_{0}^{f} \operatorname{div} \delta u^{f}=0 \\
\rho_{0}^{f} \partial_{t} \delta u^{f}=-\operatorname{grad} \delta p+\lambda \operatorname{grad}\left(\operatorname{div} \delta u^{f}\right)+2 \mu \operatorname{div} \boldsymbol{e}\left(\delta \boldsymbol{u}^{f}\right) \\
C_{p} \rho_{0}^{f} \partial_{t} \delta \theta-\theta_{0} \alpha \partial_{t} \delta p-K \Delta \theta=0 \\
\delta p=\frac{\delta \rho^{f}}{\rho_{0}^{f} \kappa_{T}}+\frac{\alpha \delta \theta}{\kappa_{T}}
\end{array}
$$

Equation (3) follows from the combination of the linearized energy conservation equation (for example [10]) with the linearized form $\delta \mathfrak{h}=C_{p} \delta \theta+\frac{1-\theta_{0} \alpha}{\rho_{0}^{f}} \delta p$ of the enthalpy equation $\mathfrak{h}=\mathfrak{h}(p, \theta)$. Equation (4) is the linearized form of the fluid state equation $p=p\left(\rho^{f}, \theta\right)$.

Let $\delta g(t)$ denote the small amplitude variations of the normal force density on the boundary $\Gamma^{s}$. Let $\boldsymbol{n}$ (resp. $\boldsymbol{t}$ ) denote the unit outer normal (resp. tangential) vector to $\Gamma^{s}$. The tangential component of the fluid velocity on $\Gamma^{s}$ vanishes. The fluid verifies the no-slip condition on $\Gamma^{f}$. The temperature is set at $\theta_{0}$ 
along the boundary, so that $\delta \theta=0$. The linearized boundary conditions read:

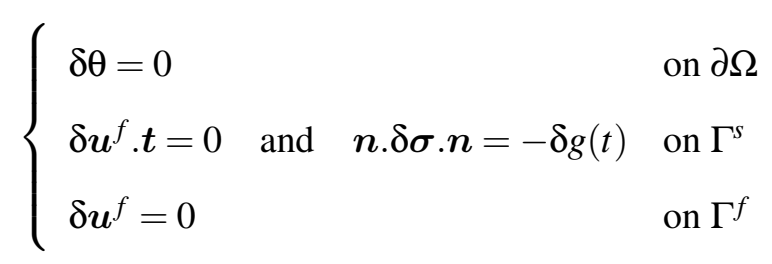

\subsubsection{Non-dimensional transformation}

Let us write $\tilde{p}=\frac{\delta p}{p_{0}}, \tilde{\boldsymbol{\rho}}^{f}=\frac{\delta \rho^{f}}{\rho_{0}^{f}}, \tilde{\theta}=\frac{\delta \theta}{\theta_{0}}, \tilde{t}=\frac{t c}{l_{x}}, \tilde{\boldsymbol{u}}^{f}=\frac{\delta \boldsymbol{u}^{f}}{c}, \tilde{\boldsymbol{\sigma}}=\frac{\delta \sigma}{\rho_{0}^{f} c^{2}}, \tilde{\boldsymbol{x}}=\frac{x}{l_{x}}, \tilde{y}=\frac{y}{l_{x}}, \tilde{z}=\frac{z}{l_{z}}, \tilde{l}_{x}=\frac{l_{x}}{l_{x}}=1$, $\tilde{l}_{y}=\frac{l_{y}}{l_{x}}, \tilde{l}_{z}=\frac{l_{z}}{l_{x}}, \tilde{\operatorname{grad}}=l_{x} \operatorname{grad}, \tilde{\operatorname{div}}=\boldsymbol{I d}: \mathbf{g r a d}, \tilde{\Delta}=\tilde{\operatorname{div}} \operatorname{grad}$ and $\tilde{\boldsymbol{e}}=\frac{1}{2}\left(\tilde{\operatorname{grad}}+\operatorname{grad}^{t}\right)$.

After substitution of the dimensional parameters in the equations and elimination of $\tilde{\rho}^{f}$ using the equation 4 , the initial-boundary value problem reads:

$$
\begin{cases}\partial_{\tilde{t}} \tilde{\boldsymbol{p}}-B \partial_{\tilde{t}} \tilde{\boldsymbol{\theta}}+A \operatorname{div} \tilde{\boldsymbol{u}}^{f}=0 & \text { on } \tilde{\Omega} \\ \partial_{\tilde{t}} \tilde{\boldsymbol{u}}^{f}+\frac{1}{M^{2}} \tilde{\operatorname{grad}} \tilde{p}-\frac{1}{R e_{2}} \operatorname{grad}\left(\tilde{\operatorname{div}} \tilde{\boldsymbol{u}}^{f}\right)-\frac{2}{R e} \tilde{\operatorname{div}} \tilde{\boldsymbol{e}}\left(\tilde{\boldsymbol{u}}^{f}\right)=0 & \text { on } \tilde{\Omega} \\ \partial_{\tilde{t}} \tilde{\boldsymbol{\theta}}-\frac{1}{C} \partial_{\tilde{t}} \tilde{\boldsymbol{p}}-\frac{1}{P e} \tilde{\Delta} \tilde{\boldsymbol{\theta}}=0 & \text { on } \tilde{\Omega} \\ \tilde{\boldsymbol{\theta}}=0 & \text { on } \partial \tilde{\Omega} \\ \tilde{\boldsymbol{u}}^{f} \cdot \boldsymbol{t}=0 \quad \text { and } \boldsymbol{n} \cdot \tilde{\boldsymbol{\sigma}} \cdot \boldsymbol{n}=-\tilde{g}_{x}(t)=-\frac{\delta g_{x}(t)}{\rho_{0}^{f} c^{2}} & \text { on } \tilde{\Gamma}^{s} \\ \tilde{\boldsymbol{u}}^{f}=0 & \text { on } \tilde{\Gamma}^{f} \\ + \text { initial conditions } & \end{cases}
$$

The definitions and values of the non-dimensional coefficients follow: $R e=\frac{c l_{x} \rho_{0}^{f}}{\mu}=1500, R_{2}=$ $\left.\frac{c l_{x} \rho_{0}^{f}}{\lambda}=-2200, M=c \sqrt{\left(\frac{\rho_{0}^{f}}{p_{0}}\right.}\right)=150, P e=\frac{l_{x} c C_{P} \rho_{0}^{f}}{K}=1.0 \times 10^{4}, A=\frac{1}{p_{0} \kappa_{T}}=2.2 \times 10^{4}, B=A * \alpha T_{0}=$ $1.3 \times 10^{3}$ and $C=\frac{C_{p} \rho_{0}^{f}}{\alpha p_{0}}=2.0 \times 10^{5}$.

Note that $R e$ is not the usual Reynolds number, because it does not depend on the actual fluid velocity. Let $R e_{u}$ denote the usual Reynolds number. A relevant definition of $R e_{u}$ for the considered system could be $R e_{u}=\frac{R e Y_{\max } \omega}{c}$, where $Y_{\max }$ stands for the maximal amplitude of displacement of the vibrating microplate and $\omega$ for the angular frequency. For vibrations of amplitude $Y_{\max }=1 \mathrm{~nm}$ in water between $10 \mathrm{MHz}$ and $2 \mathrm{GHz}, R e_{u}$ verifies $0.06<R e_{u}<12$. 


\subsubsection{Neglecting the temperature variations}

In water, the parameter $C$ verifies $C>>1$. Considering the respective orders of magnitude of the time derivatives appearing in the system 6 , the term $\frac{1}{C} \partial_{\tilde{t}} \tilde{p}$ can be neglected: the energy equation depends only on $\tilde{\theta}$ and can be solved regardless of the other parameters. So let us now assume that the temperature variation $\tilde{\theta}$ verifies $\tilde{\theta}=0$ on the boundary $\partial \tilde{\Omega}$ and $\tilde{\theta}(\tilde{t}=0)=0$ on $\tilde{\Omega}$. Solving the energy equation with $C^{-1}=0$ leads to $\tilde{\theta}=0$ on $\tilde{\Omega}$ for all time $\tilde{t}>0$. Note that in dry air, this conclusion would not apply because $C=3.5 \times 10^{-6}<<1$.

In the rest of the paper, we assume that $\tilde{\theta}=0$ on $\tilde{\Omega}$ for all time $\tilde{t}>0$. The initial-boundary value problem to be solved writes:

$$
\left\{\begin{array}{lr}
\partial_{\tilde{t}} \tilde{\boldsymbol{p}}+A \operatorname{div} \tilde{\boldsymbol{u}}^{f}=0 & \text { on } \tilde{\Omega} \\
\partial_{\tilde{t}} \tilde{\boldsymbol{u}}^{f}+\frac{1}{M^{2}} \tilde{\operatorname{grad}} \tilde{p}-\frac{1}{R e_{2}} \tilde{\operatorname{grad}}\left(\operatorname{div} \tilde{\boldsymbol{u}}^{f}\right)-\frac{2}{R e} \tilde{\operatorname{div}} \tilde{\boldsymbol{e}}\left(\tilde{\boldsymbol{u}}^{f}\right)=0 & \text { on } \tilde{\Omega} \\
\tilde{\boldsymbol{u}}^{f} \cdot \boldsymbol{t}=0 \text { and } \boldsymbol{n} \cdot \tilde{\boldsymbol{\sigma}}\left(\tilde{p}, \tilde{\boldsymbol{u}}^{f}\right) \cdot \boldsymbol{n}=-\tilde{g}_{x}(t) & \text { on } \tilde{\Gamma}^{s} \\
\tilde{\boldsymbol{u}}^{f}=0 & \text { on } \tilde{\Gamma}^{f} \\
+ \text { initial conditions } &
\end{array}\right.
$$

\subsection{Structural equations}

\subsubsection{Boundary value problem}

Assuming that the plate is isotropic, sufficiently thin and clamped along $\partial \Gamma^{s}[31]$, its equation of motion reads

$$
\begin{cases}\rho^{s} h \partial_{t t} d^{s}+\Delta\left(D \Delta d^{s}\right)=g & \text { on } \Gamma^{s} \\ d^{s}=0 \text { and } \operatorname{grad} d^{s} \cdot \boldsymbol{n}=0 & \text { on } \partial \Gamma^{s} \\ + \text { initial conditions } & \end{cases}
$$

where $d^{s}$ is the transverse displacement field of the plate in the $x$ direction, $\Delta=\partial_{y y}+\partial_{z z}$ the Laplace operator, $D=\frac{E h^{3}}{12\left(1-v^{2}\right)}$ the scalar bending stiffness of the plate, $E$ its Young's modulus, $h$ its thickness, $\rho^{s}$ its density, $v$ its Poisson's ratio, $g$ the surface force density applied to the plate. Its temperature is homogeneous and is equal to $\theta_{0}$.

For the targeted application, the plate is a dense assembly of one, or very few layers, of aligned single walled carbon nanotubes [5]. We use parameter values typical of those of single walled carbon 
nanotubes: $E=1 \mathrm{TPa}$ [32], $h=1 \mathrm{~nm}, \rho^{s}=2000 \mathrm{~kg} \cdot \mathrm{m}^{-3}$. In the absence of experimental data, we set $v=0.3$. Of course the following numerical methodology remains valid for a large range of plate parameters.

The actuation of the plate results from the capacitive force exerted on the metallic plate by the actuation electrode under time-varying voltage. The actuation force density $g$ is theoretically proportional to $V(t)^{2} \frac{\partial \mathcal{C}}{\partial d^{s}}$ [33], where $V(t)$ is the time-varying voltage applied to the electrode and $\mathcal{C}\left(d^{s}, R\right) \propto \frac{1}{R+d^{s}}$ is the capacitance between the plate and the electrode, which depends on the plate displacement $d^{s}$ and on the plate-electrode distance $R$.

As a first step toward taking into account the exact actuation force, we assume the force density applied by the electrode on the plate to take the form $g=g_{0}(t)+g_{1} d^{s}(t)=G_{0} \cos (\omega t)+g_{1} d^{s}(t)$, with $\omega$ the angular frequency and $\left|d^{s}\right|<<R$. The sign of $g_{1}$ is opposite to the sign of $G_{0}$, due to the first order Taylor expansion of the term $\frac{\partial \mathcal{C}}{\partial d^{s}}$ for $\left|d^{s}\right|<<R$. We set $G_{0}=-8000 \mathrm{~Pa}$ and $g_{1}=0.16 \mathrm{TPa}^{-1}$, which is the order of magnitude of the force density applied by an electrode under $2.5 \mathrm{~V}$ located at $100 \mathrm{~nm}$ of the plate.

\subsubsection{Non-dimensional transformation}

Reference parameters for the non-dimensional transformation of this plate boundary value problem are $c, \rho_{0}^{f}$ and $l_{x}$. Assuming the plate is homogeneous, problem 8 transforms into

$$
\begin{cases}a_{p} \partial_{\tilde{t} \tilde{t}} \tilde{d}^{s}+r_{p} \tilde{\Delta} \tilde{\Delta} \tilde{d}^{s}=\tilde{g} & \text { on } \tilde{\Gamma}^{s} \\ \tilde{d}^{s}=0 \text { and } \operatorname{grad} \tilde{d}^{s} . n=0 & \text { on } \partial \tilde{\Gamma}^{s} \\ + \text { initial conditions } & \end{cases}
$$

where $\tilde{d}^{s}=\frac{d^{s}}{l_{x}}, \tilde{x}=\frac{x}{l_{x}}, \tilde{y}=\frac{y}{l_{x}}, \tilde{t}=\frac{t c}{l_{x}}, \tilde{\Delta}=\frac{\Delta}{l_{x}}, \tilde{g}=\frac{g}{\rho_{0}^{f} c^{2}}, \tilde{g}_{0}=\frac{g_{0}}{\rho_{0}^{f} c^{2}}, \tilde{g}_{1}=\frac{g_{1} l_{x}}{\rho_{0}^{f} c^{2}}, a_{p}=\frac{h \rho^{s}}{l_{x} \rho_{0}^{f}}$ and $r_{p}=$ $\frac{D}{l_{x}^{3}}=\frac{E}{\rho_{0}^{f} c^{2}} \frac{h^{3}}{12 l_{x}^{3}\left(1-v^{2}\right)}$. To be short, foremost validity conditions are $h<<l_{x}$ et $\tilde{d}^{s}<<1$. Typical values for these non-dimensional parameters are $r_{p}=4.1 \times 10^{-8}, a_{p}=2.0 \times 10^{-3}, \tilde{g}_{1}=7.3 \times 10^{-5}$ and $\tilde{g}_{0}=$ $\left(3.6 \times 10^{-6}\right) \cos (\omega t)$. 


\subsection{Fluid structure interaction}

\subsubsection{Coupling equations}

In the rest of this paper, unless specifically mentioned, we consider only non-dimensional quantities. Hence, for the sake of simplicity, from now on, we drop the symbol in all equations.

According to the perfect kinematic continuity assumption and associated stress continuity condition on $\Gamma^{s}$, the fluid-structure problem is governed by the following boundary value problem:

$$
\begin{cases}\partial_{t} p+A \operatorname{div} \boldsymbol{u}^{f}=0 & \text { on } \Omega \\ \partial_{t} \boldsymbol{u}^{f}=-\frac{1}{M^{2}} \operatorname{grad} p+\frac{1}{R e_{2}} \operatorname{grad} \operatorname{div} \boldsymbol{u}^{f}+\frac{2}{R e} \operatorname{div} \boldsymbol{e}\left(\boldsymbol{u}^{f}\right) & \text { on } \Omega \\ \boldsymbol{u}^{f}=0 & \text { on } \Gamma^{f} \\ u_{y}^{f}=0 \quad \text { and } \quad u_{x}^{f}=\partial_{t} d^{s} & \text { on } \Gamma^{s} \\ g_{0}+\boldsymbol{n} \cdot\left(\left(-\frac{1}{M^{2}} p+\frac{1}{R e_{2}} \operatorname{div} \boldsymbol{u}^{f}\right) \boldsymbol{I} \boldsymbol{d}+\frac{2}{R e} \boldsymbol{e}\left(\boldsymbol{u}^{f}\right)\right) \cdot \boldsymbol{n}=-g_{1} d^{s}+a_{p} \partial_{t t} d^{s}+r_{p} \Delta \Delta d^{s} & \text { on } \Gamma^{s} \\ d^{s}=0 \quad \text { and } \operatorname{grad} d^{s} \cdot \boldsymbol{n}=0 & \text { on } \partial \Gamma^{s} \\ + \text { initial conditions } & \end{cases}
$$

In view of writing the variational formulation of the coupled problem, it is convenient to reformulate the dynamics of the structure as a first-order system using displacement, pressure and velocity as unknowns. To this end, let $u^{s}$ stand for the non-dimensional transverse velocity field in the solid. As usual, the dynamics rewrites

$$
\begin{cases}\partial_{t} d^{s}-u^{s}=0 & \text { on } \Gamma^{s} \\ -g_{1} d^{s}+a_{p} \partial_{t} u^{s}+r_{p} \Delta \Delta d^{s}-\sigma\left(p, \boldsymbol{u}^{f}\right) \cdot \boldsymbol{n}=g_{0} & \text { on } \Gamma^{s}\end{cases}
$$

Note that following [31] the last equation rewrites

$$
a_{p} \partial_{t} u^{s}+r_{p} \Delta \Delta\left(\int_{0}^{t} u^{s}\right)-g_{1} \int_{0}^{t} u^{s}-\sigma\left(p, u^{f}\right) \cdot \boldsymbol{n}=g_{0} \text { on } \Gamma^{s}
$$

and, assuming the structural velocity is known, the displacement can be obtained as $d^{s}=\int_{0}^{t} u^{s}$ 


\subsubsection{Variational formulation}

Let $u=\left\{u^{f}, p, u^{s}\right\}$ denote the triplet formed by the non-dimensional velocity and pressure fields in the fluid, and by the non-dimensional velocity field in the solid respectively. Following [31], the natural solution space writes

$$
\mathbb{V}=\left\{v=\left\{v^{f}, r, v^{s}\right\} \in\left(H^{1}\left(\Omega_{f}\right)\right)^{3} \times L^{2}\left(\Omega_{f}\right) \times H_{0}^{2}\left(\Gamma_{s}\right) ; v^{f} . \boldsymbol{n}=v^{s} \text { and } v^{f} . \boldsymbol{t}=0 \text { on } \Gamma^{s} ; v^{f}=0 \text { on } \Gamma^{f}\right\}
$$

where $H_{0}^{2}\left(\Gamma^{s}\right)=\left\{v \in H^{2}\left(\Gamma^{s}\right) \mid d^{s}=0\right.$ and $\operatorname{grad} d^{s} . \boldsymbol{n}=0$ on $\left.\partial \Gamma^{s}\right\}$

This is well-defined since the trace of the velocity field along $\Gamma^{s}$ is a square-summable function. Moreover, the space associated with inertia writes $H=L^{2}\left(\Omega^{f}\right)^{3} \times L^{2}\left(\Gamma^{s}\right)$.

Let us introduce the bilinear form that corresponds to the virtual work of internal stresses

$$
\begin{array}{r}
a(u, v)=\int_{\Omega^{f}} \frac{M^{2}}{R e_{2}}\left(\operatorname{div} u^{f}\right)\left(\operatorname{div} v^{f}\right)+\frac{2 M^{2}}{R e} e\left(u^{f}\right): e\left(v^{f}\right)+\int_{\Omega^{f}}\left(\operatorname{div} u^{f}\right) r-p \operatorname{div} v^{f} \\
+\frac{r_{p}}{a_{p}} \int_{\Gamma^{s}} \Delta\left(\int_{0}^{t} u^{s}\right) \Delta v^{s}-\frac{g_{1}}{a_{p}} \int_{\Gamma^{s}}\left(\int_{0}^{t} u^{s}\right) v^{s} \quad \forall\left(u=\left\{u^{f}, p, u^{s}\right\}, v=\left\{v^{f}, r, v^{s}\right\}\right) \in \mathbb{V}^{2}
\end{array}
$$

The bilinear form associated with the acceleration reads

$$
b(u, v)=\int_{\Omega^{f}}\left(M^{2} u^{f} v^{f}+\frac{1}{A} p r\right)+\int_{\Gamma^{s}} u^{s} v^{s} \quad \forall\left(u=\left\{u^{f}, p, u^{s}\right\}, v=\left\{v^{f}, r, v^{s}\right\}\right) \in \mathbb{V}^{2}
$$

The plate will be subjected to an external distributed force $g_{0}$ and there is no other external force on the system. Let us call $L(v)=\int_{\Gamma^{s}} g_{0} v^{s} \quad \forall v=\left\{v^{f}, r, v^{s}\right\} \in \mathbb{V}$.

It is a purely computational and standard matter to show that the fluid-structure coupled problem writes

$$
\begin{cases}\text { seek } \quad u \in L^{2}(0, T ; V) & \text { such that } \\ b\left(\partial_{t} u, v\right)+a(u, v)=L(v) & \forall v \in \mathbb{V}\end{cases}
$$

The well-posedness of this problem relies on a variant of general arguments of the energy method such as those explained in [31] (vol 5, chapter 18, pages 670-675). More precisely, the following existence and uniqueness result can be established: 
Existence and uniqueness of solutions : Assume the spring stiffness $g_{1}$ to be smaller than the first eigenvalue of the clamped micro-plate. Then there exists a unique solution $u=\left\{u^{f}, p, u^{s}\right\} \in L^{\infty}(0, T ; H)$ of equation (16) such that $\left\{u^{f}, p, \int_{0}^{t} u^{s}\right\} \in \mathbb{V}$. In particular, $u^{f} \in L^{2}\left(0, T ; H^{1}\left(\Omega^{f}\right)\right), p \in L^{2}\left(0, T ; L^{2}\left(\Omega^{f}\right)\right)$ and $\int_{0}^{t} u^{s} \in L^{\infty}\left(0, T ; H_{0}^{2}\left(\Gamma^{s}\right)\right)$. As a result, the structural displacement $d^{s}=\int_{0}^{t} u^{s} \in L^{\infty}\left(0, T ; H_{0}^{2}\left(\Gamma^{s}\right)\right)$ is bounded in time and space and the no-jump condition of the velocity field along the fluid-structure interface $\Gamma^{s}$ takes place almost everywhere.

\subsubsection{Forced response under harmonic load}

The external force is harmonic: $g_{0}=G_{0} \cos (\omega t)$ where $\omega$ is the reduced (e.g. non-dimensional) angular frequency such as the actual angular frequency (expressed in $\mathrm{rad}^{-1} \mathrm{~s}^{-1}$ ) is equal to $\omega_{l_{x}}$. If $f$ is a given non-dimensional scalar or vector field, let $F$ denote its Fourier transform. Writing $q=i \omega$ and $U=\left\{U^{f}, P, U^{s}\right\}$, one has to solve the following set of equations:

$$
\begin{cases}q P+A \operatorname{div} \boldsymbol{U}^{f}=0 & \text { on } \Omega \\ q \boldsymbol{U}^{f}+\frac{1}{M^{2}} \operatorname{grad} P-\frac{1}{R e_{2}} \operatorname{grad} \operatorname{div} \boldsymbol{U}^{f}-\frac{2}{R e} \operatorname{div} \boldsymbol{e}\left(\boldsymbol{U}^{f}\right)=0 & \text { on } \Omega \\ \boldsymbol{U}^{f}=0 & \text { on } \Gamma^{f} \\ U_{y}^{f}=0 \quad \text { and } \quad U_{x}^{f}=q D^{s} & \text { on } \Gamma^{s} \\ G_{0}+\boldsymbol{n} \cdot\left(\left(-\frac{1}{M^{2}} P+\frac{1}{R e_{2}} \operatorname{div} \boldsymbol{U}^{f}\right) \boldsymbol{I} \boldsymbol{d}+\frac{2}{R e} \boldsymbol{e}\left(\boldsymbol{U}^{f}\right)\right) \cdot \boldsymbol{n}=\frac{-g_{1}+a_{p} q^{2}}{q} U^{s}+\frac{r_{p}}{q} \Delta \Delta D^{s} & \text { on } \Gamma^{s} \\ D^{s}=0 \quad \text { and } \quad \operatorname{grad} D^{s} \cdot \boldsymbol{n}=0 & \text { on } \partial \Gamma^{s}\end{cases}
$$

A variational formulation of previous set of equations now reads

$$
\begin{cases}\text { seek } \quad U \in \mathbb{V} & \text { such that } \\ q b(U, \bar{V})+a(U, \bar{V})=\int_{\Gamma^{s}} G_{0} \bar{U}^{S} & \forall V \in \mathbb{V}\end{cases}
$$

Where the time integrals are to be replaced with the multiplicative factor $\frac{1}{i \omega}$. It is easy to prove that no $q=i \omega$ is an eigenvalue of above problem, that is to say, any solution of this problem should vanish when the applied force vanishes. Such a solution $U$ would satisfy above equation with $V=U$, thus 


$$
b(U, U)-\frac{r_{p}}{a_{p}} \int_{\Gamma^{s}} \Delta U^{s} \Delta U^{s}=0 \quad \int_{\Omega^{f}} \frac{M^{2}}{R e_{2}}\left(\operatorname{div} U^{f}\right)\left(\operatorname{div} \bar{U}^{f}\right)+\frac{2 M^{2}}{\operatorname{Re}} e\left(U^{f}\right): e\left(\bar{U}^{f}\right)=0
$$

Korn's inequality together with homogeneous Dirichlet boundary conditions on $\Gamma^{f}$ thus lead to $U^{f}=$ 0 over $\Omega$. Because of the kinematic transmission condition between the fluid and the structure, we get $U^{s}=0$ over $\Gamma^{s}$. The first part of above equation then leads to $P=0$ over $\Omega$. The same coerciveness argument enables one to establish the well-posedness of problem (18).

\subsubsection{Condensation of the fluid problem on the structure}

Let $\mathcal{N}$ denote the Neumann extension operator from $L^{2}\left(\Gamma^{s}\right)$ to $L^{2}(\Omega) \times H^{1}(\Omega)^{3}$ defined as

$$
\begin{aligned}
& \mathcal{N} \quad: \quad L^{2}\left(\Gamma^{s}\right) \longrightarrow L^{2}(\Omega) \times H^{1}(\Omega)^{3} \\
& G \quad \longrightarrow\left(P, U_{x}^{f}, U_{y}^{f}\right) \\
& \begin{array}{lll}
\text { such that } & \frac{q}{A} P+\operatorname{div} U^{f}=0 & \text { on } \Omega
\end{array} \\
& q \boldsymbol{U}^{f}+\frac{1}{M^{2}} \operatorname{grad} P-\frac{1}{R e_{2}} \operatorname{grad} \operatorname{div} \boldsymbol{U}^{f}-\frac{2}{R e} \operatorname{div} \boldsymbol{e}\left(\boldsymbol{U}^{f}\right)=0 \quad \text { on } \Omega \\
& \begin{array}{ll}
\boldsymbol{U}^{f}=0 & \text { on } \Gamma^{f}
\end{array} \\
& \begin{array}{ll}
U_{y}^{f}=0 & \text { on } \Gamma^{s}
\end{array} \\
& \boldsymbol{n} \cdot\left(\left(-\frac{1}{M^{2}} P+\frac{1}{R e_{2}} \operatorname{div} \boldsymbol{U}^{f}\right) \boldsymbol{I} \boldsymbol{d}+\frac{2}{R e} \boldsymbol{e}\left(\boldsymbol{U}^{f}\right)\right) \cdot \boldsymbol{n}=-G \quad \text { on } \Gamma^{s}
\end{aligned}
$$

Let $\mathcal{T} r$ denote the trace on $\Gamma^{s}$ of $U_{x}^{f}$. Let us then define $S$ by $S=\mathcal{T} r \circ \mathcal{N}$. Let $\mathcal{P}$ denote the following unbounded operator (for $q \neq 0$ ):

$$
\begin{aligned}
\mathcal{P}: H_{0}^{2}\left(\Gamma^{s}\right) & \longrightarrow L^{2}\left(\Gamma^{s}\right) \\
U^{s} & \longrightarrow \frac{a_{p} q^{2}-g_{1}}{q} U^{s}+\frac{r_{p}}{q} \Delta \Delta U^{s}
\end{aligned}
$$

Let $G_{0} \in L^{2}\left(\Gamma^{s}\right)$ denote the Fourier transform of the non-dimensional load $g_{0}$ applied on the plate by the fluid. Combining the definition of $\mathcal{S}$ with equations (17), (20) and (21), the coupled system thus reads:

$$
\mathcal{P}\left(U^{s}\right)+\left(\mathcal{S}^{-1}\right)\left(U^{s}\right)=G_{0}
$$


Writing $\mathcal{T}=\mathcal{S}^{-1}$, the variational formulation of problem (22) reads, :

$$
\begin{cases}\text { seek } U^{s} \in H_{0}^{2}\left(\Gamma^{s}\right) & \text { such that } \\ \int_{\Gamma^{s}}\left(\mathcal{P}\left(U^{s}\right) \bar{Z}+\mathcal{T}\left(U^{s}\right) \bar{Z}\right)=\int_{\Gamma^{s}} G_{0} \bar{Z} & \forall Z \in H_{0}^{2}\left(\Gamma^{s}\right)\end{cases}
$$

where above integrals should be understood as the duality product $\left.H^{-2}<\right\rangle_{H_{0}^{2}}$.

A motivation for condensing the fluid problem lies in the wish to investigate the behaviour of the actuator itself. The method is expected to be especially useful in the field of micro- and nanoelectromechanical systems modeling, because, at this scale, structure equations are more complex due to downscaling.

The proposed formulation also lays the foundations for the use of independent, special purpose solvers for the fluid and the structure. In particular, it may be computationally efficient to do so in the three-dimensional case, especially when the microplate behaves in its non linear regime. However, it is presently unclear how this choice will impact further studies on the observability of the characteristics of the fluid domain.

\subsection{Approximate problem}

\subsubsection{Choice of the approximation spaces}

Let $\mathbb{W}_{N}$ denote the span of a basis of $\left(L^{2}\left(\Gamma^{s}\right)\right)^{2}$, for instance the Fourier basis $\mathcal{F}_{N}=$ $\left(f_{n, m}^{i}\right)_{(i, n, m) \in \llbracket 1,4 \rrbracket \times \llbracket 0, N \rrbracket \times \llbracket 0, N \rrbracket}$ with $\mathcal{M}_{3 D}=4 N^{2}+4 N+1$ elements (only keeping non identically vanishing functions) defined as follows:

$$
\begin{gathered}
f_{n, m}^{1}(x, y)=\cos \left(2 \pi n \frac{x}{l_{x}}\right) \cos \left(2 \pi m \frac{y}{l_{y}}\right), \quad f_{n, m}^{2}(x, y)=\cos \left(2 \pi n \frac{x}{l_{x}}\right) \sin \left(2 \pi m \frac{y}{l_{y}}\right), \\
f_{m, n}^{3}(x, y)=\sin \left(2 \pi n \frac{x}{l_{x}}\right) \cos \left(2 \pi m \frac{y}{l_{y}}\right) \quad \text { and } \quad f_{n, m}^{4}(x, y)=\sin \left(2 \pi n \frac{x}{l_{x}}\right) \sin \left(2 \pi m \frac{y}{l_{y}}\right)
\end{gathered}
$$

Let us define $\tau_{0}(x, y)=x^{2}\left(l_{x}-x\right)^{2} y^{2}\left(l_{y}-y\right)^{2}$ and $\mathbb{V}_{N}$ the span of the set $\mathcal{G}_{N}=$ $\left(\tau_{0} f_{n, m}^{i}\right)_{(i, n, m) \in \llbracket 1,4 \rrbracket \times \llbracket 0, N \rrbracket \times \llbracket 0, N \rrbracket}$. The projection from $L_{2}$ to $\mathbb{V}_{N}$ is denoted $\Pi_{N}$.

The set of functions $\left\{\left(x^{i} y^{j} \tau_{0}(x, y)\right)_{0 \leq i, j \leq+\infty}\right\}$ generates $H_{2}^{0}\left(\Gamma^{s}\right)$ because $\tau_{0}$ divides any polynomial $v$ verifying $v=0$ and grad $v \cdot n=0$ on $\partial \Gamma^{s}$. As $\mathcal{F}_{\infty}$ generates $L_{2}\left(\Gamma^{s}\right)$, it also generates $\operatorname{Span}\left\{\left(x^{i} y^{j}\right)_{0 \leq i \leq+\infty ; 0 \leq j \leq+\infty}\right\}$. Hence, $\mathcal{G}_{\infty}$ generates $H_{2}^{0}\left(\Gamma^{s}\right)$, so that we choose $\mathbb{V}_{N}$ as approximation space 
for $H_{0}^{2}\left(\Gamma^{s}\right)$.

The interest of using $\mathbb{V}_{N}$ as approximation space for $H_{2}^{0}\left(\Gamma^{s}\right)$ instead of $\operatorname{Span}\left\{\left(x^{i} y^{j} \tau_{0}(y)\right)_{0 \leq i, j \leq+\infty}\right\}$ lies in computational issues. The interest of using the set of functions $\mathbb{V}_{N}$ instead of a finite elements space lies in the fact that spectral methods are known to converge exponentially fast. They are usually limited to polyhedral volumes where they are easier to implement. Since we consider here a rectangular plate, this method will prove efficient. If we were to consider a plate of arbitrary shape, we would use finite elements.

As a complement, let us briefly define an appropriate set of functions $\mathcal{H}_{N}$ for the 2D setting: writing $\psi_{0}(x)=x^{2}\left(l_{x}-x\right)^{2}, \mathcal{H}_{N}$ is defined as the set of $\mathcal{M}_{2 D}=2 N+1$ functions:

$$
\forall n \in \llbracket 0, N \rrbracket \quad \psi_{n}^{1}(x)=\psi_{0}(x) \cos \left(2 \pi n \frac{x}{l_{x}}\right) \quad \text { and } \quad \forall n \in \llbracket 1, N \rrbracket \quad \psi_{n}^{2}(x)=\psi_{0}(x) \sin \left(2 \pi n \frac{x}{l_{x}}\right)
$$

\subsubsection{Approximation of operators and approximate resolution}

The approximation of coupled problem $(23)$ on $\mathbb{V}_{N}$ reads:

$$
\begin{cases}\text { seek } U^{s} \in \mathbb{V}_{N} & \text { such that } \\ \int_{\Gamma^{s}}\left(\mathcal{P}\left(U^{s}\right) \bar{Z}+\mathcal{T}\left(U^{s}\right) \bar{Z}\right)=\int_{\Gamma^{s}} G_{0} \bar{Z} & \forall Z \in \mathbb{V}_{N}\end{cases}
$$

This corresponds to the following approximations $\mathcal{P}_{N}, \mathcal{T}_{N}, G_{0, N}$ of the operators $\mathcal{P}$ and $\mathcal{T}$ and of the external distributed force $G_{0}$ :

$$
\mathcal{P}_{N}=\Pi_{N} \mathcal{P} \Pi_{N}, \quad \mathcal{T}_{N}=\Pi_{N} \mathcal{T} \Pi_{N}, \quad G_{0, N}=\Pi_{N} G_{0}
$$

In terms of these approximate operators, the approximate problem reads

$$
\text { seek } U^{s} \in \mathbb{V}_{N} \text { such that } \mathcal{P}_{N}\left(U^{s}\right)+\mathcal{T}_{N}\left(U^{s}\right)=G_{0, N}
$$

Let us write, with $\mathcal{M}=\mathcal{M}_{3 D}$ in the $3 \mathrm{D}$ case and $\mathcal{M}=\mathcal{M}_{2 D}$ in the $2 \mathrm{D}$ case,

$$
\begin{gathered}
U^{s}=\sum_{i=1}^{\mathcal{M}} u_{i}^{s} \tau_{i}, \quad U_{e}^{s}=\left(u_{i}^{s}\right)_{1 \leq i \leq \mathcal{M}}, \quad G_{e}=\left(<\tau_{i}, G_{0}>\right)_{1 \leq i \leq \mathcal{M}}, \quad P_{e}=\left(<\tau_{i}, \mathcal{P}\left(\tau_{j}\right)>\right)_{1 \leq i, j \leq \mathcal{M},}, \\
N_{e}=\left(<f_{i}, f_{j}>\right)_{1 \leq i, j \leq \mathcal{M}}, \quad S_{e}=\left(<f_{i}, S\left(f_{j}\right)>\right)_{1 \leq i, j \leq \mathcal{M}} \quad \text { and } \quad T_{e}=\left(<f_{i}, \mathcal{T}\left(f_{j}\right)>\right)_{1 \leq i, j \leq \mathcal{M}}
\end{gathered}
$$


Let us recall that

$$
<\tau_{j}, \mathcal{P}\left(\tau_{i}\right)>=\left(f_{1}-a \omega^{2}\right)<\tau_{i}, \tau_{j}>+d<\Delta \tau_{i} \Delta \tau_{j}>\quad \text { and } \quad T_{e}=N_{e} S_{e}^{-1} N_{e}
$$

The matrix form of problem (28) reads:

$$
\left(P_{e}+N_{e} S_{e}^{-1} N_{e}\right) U_{e}^{s}=G_{e}
$$

According to paragraph 3.4.3, the matrix $P_{e}+N_{e} S_{e}^{-1} N_{e}$ is inversible. The matrix equation (29) has a unique solution $U_{e}^{s}$, defining the unique solution $U^{s} \in \mathbb{V}_{N}$ of the approximate problem (28).

\section{Numerical study of the convergence of the approximate problem}

\subsection{Implementation of the computational method}

The operators $\mathcal{N}$ and $\mathcal{S}$ the trace of $\mathcal{N}$ on $\Gamma^{s}$ are evaluated by a standard finite element method with the software FreeFem++ (version 2.19) [34].

In 2D, we use a regular mesh made of triangles generated by FreeFem++, in 3D, a tetrahedral mesh generated by TetGen [35]. The mesh density is controlled by the number $n$ of nodes along the edge $\{y=0, z=0,0<x<1\}$, which we call here the 'mesh resolution'. The number of nodes by unit length is the same for all edges, so that the total number of nodes in the domain $\Omega$ is proportional to $n^{2}$ in $2 \mathrm{D}$, $n^{3}$ in $3 \mathrm{D}$.

The finite elements used for the pressure field $P$ are P1-Lagrange (continuous piecewise linear finite elements) and the ones for the velocity field $\boldsymbol{U}^{f} \mathrm{P} 1 \mathrm{~b}(\mathrm{P} 1+$ bubble) as defined in [36]. We use FreeFem++'s version of the UMFPACK solver [37].

Using this finite element method, we evaluate the operator $S_{N}$. In practice, we compute each finite element function $\mathcal{S}\left(f_{i}\right)$ for $i \in \llbracket 1, \mathcal{M} \rrbracket$. By a straightforward step of numerical integration under FreeFem++, we then computes the $\mathcal{M}^{2}$ coefficients of the matrix $S_{f}=\left(<f_{i}, \mathcal{S}\left(f_{j}\right)>\right)_{1 \leq i, j \leq \mathcal{M}}$. We also compute the coefficients of the other required matrices and vectors, namely $P_{e}, G_{e}$ and $N_{e}$. We use FreeFem++ version of the lapack module [38] to solve the matrix equation 29. 


\subsection{Convergence of the fluid computation}

In this section, we study the precision of the finite element method implemented to evaluate the operators $\mathcal{N}$ et $\mathcal{S}$. For this convergence study, we use the parameters $\omega=0.90, A=22000, M=150$, $R e=1500, R e_{2}=-2200, l_{x}=1, l_{y}=1$ and $l_{z}=1.3$. Let us choose a reference velocity field $\boldsymbol{U}_{0}^{f}$ :

$$
U_{0, x}^{f}=U_{0, y}^{f}=x\left(l_{x}-x\right) y\left(l_{y}-y\right) z\left(l_{z}-z\right), \quad U_{0, z}^{f}=\left(l_{z}^{2}-z^{2}\right) x\left(l_{x}-x\right) y\left(l_{y}-y\right)
$$

We derive analytically the functions $P_{0}=-\frac{A}{q} \operatorname{div} \boldsymbol{U}_{0}^{f}, \boldsymbol{f}_{0}$ and $G_{0}$ such that $\left(P_{0}, \boldsymbol{U}_{0}^{f}\right)$ verifies $\mathcal{N}_{f_{0}}\left(G_{0}\right)=\left(P_{0}, U_{0}^{f}\right)$, where $\mathcal{N}_{f_{0}}$ is the affine Neumann extension operator defined as

$$
\begin{aligned}
& \mathcal{N}_{f_{0}} \quad: \quad L^{2}\left(\Gamma^{s}\right) \longrightarrow L^{2}(\Omega) \times H^{1}(\Omega)^{3} \\
& G \quad \longrightarrow\left(P, U_{x}^{f}, U_{y}^{f}, U_{z}^{f}\right) \\
& \begin{array}{ll}
\text { such that } \quad \frac{q}{A} P+\operatorname{div} U^{f}=0 & \text { on } \Omega
\end{array} \\
& q \boldsymbol{U}^{f}+\frac{1}{M^{2}} \operatorname{grad} P-\frac{1}{R e_{2}} \operatorname{grad}\left(\operatorname{div} \boldsymbol{U}^{f}\right)-\frac{2}{R e} \operatorname{div} \boldsymbol{e}\left(\boldsymbol{U}^{f}\right)=\boldsymbol{f}_{0} \quad \text { on } \Omega \\
& U^{f}=0 \quad \text { on } \Gamma^{f}
\end{aligned}
$$

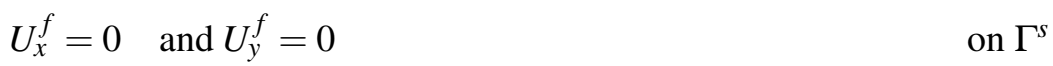

$$
\begin{aligned}
& \boldsymbol{n} \cdot\left(\left(-P+\frac{1}{R e_{2}} \operatorname{div} \boldsymbol{U}^{f}\right) \boldsymbol{I} \boldsymbol{d}+\frac{2}{R e} e\left(\boldsymbol{U}^{f}\right)\right) \cdot \boldsymbol{n}=-G \quad \text { on } \Gamma^{s}
\end{aligned}
$$

Let $\mathcal{N}_{f_{0}, n}$ denote the approximation by finite elements of the operator $\mathcal{N}_{f_{0}}$ with a mesh resolution $n$. Let $\|X\|=\left(\int_{\Omega}|X|^{2}\right)^{1 / 2}$ denote the usual $L^{2}(\Omega)$ norm and let us write $\left(P_{n}, U_{x, n}^{f}, U_{y, n}^{f}, U_{z, n}^{f}\right)=\mathcal{N}_{f_{0}, n}\left(G_{0}\right)$, $\varepsilon_{x}=\frac{\left\|U_{n, x}^{f}-U_{0, x}^{f}\right\|}{\left\|U_{0, x}^{f}\right\|}, \varepsilon_{y}=\frac{\left\|U_{n, y}^{f}-U_{0, y}^{f}\right\|}{\left\|U_{0, y}^{f}\right\|}$ and $\varepsilon_{z}=\frac{\left\|U_{n, z}^{f}-U_{0, z}^{f}\right\|}{\left\|U_{0, z}^{f}\right\|}$. By construction, we expect $\left(P_{n}, U_{x, n}^{f}, U_{y, n}^{f}, U_{z, n}^{f}\right)$ to converge toward $\left(P_{0}, \boldsymbol{U}_{0}^{f}\right)$ when $n$ increases. Indeed, Figure 2 shows the decrease of relative errors $\varepsilon_{x}$ and $\varepsilon_{z}$ toward 0 when $n$ increases (by symmetry, in this setting $\varepsilon_{x}=\varepsilon_{y}$ ). The decay rate indicates second order convergence. 


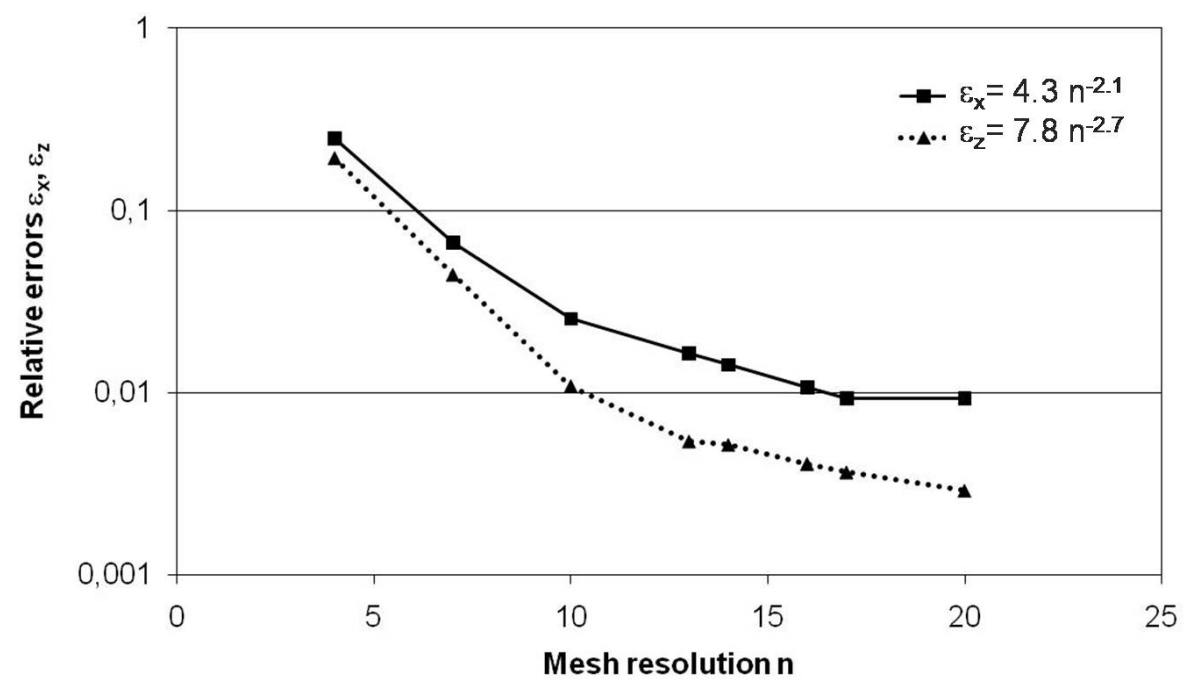

Figure 2: Convergence of the computed solution toward a known polynomial solution: relative errors $\varepsilon_{x}$ and $\varepsilon_{z}$ with respect to the mesh resolution $n$

\subsection{Convergence of the elasto-acoustic computation}

\subsubsection{Principle of the method}

In this section, we study the precision of the method used for the evaluation of $U^{s} \in \mathbb{V}_{N}$, as defined in equation (28), with respect to $N$ (the dimension of the space $\mathbb{V}_{N}$ being equal to $4 N^{2}+4 N+1$ in $3 \mathrm{D}$ and $2 N+1$ in 2D) and $n$ (the mesh resolution). The parameters used for the fluid equations are the same as in the previous paragraph. The parameters used for the plate equation are $r_{p}=4.1 \times 10^{-8}, a_{p}=2.0 \times 10^{-3}$, $G_{0}=3.6 \times 10^{-6}$ and $g_{1}=7.3 \times 10^{-5}$.

Like in the previous paragraph, we choose a reference velocity field $\boldsymbol{U}_{0}^{f}$ from which we derive analytically the functions $P_{0}=-\frac{A}{q} \operatorname{div} \boldsymbol{U}_{0}^{f}, \boldsymbol{f}_{0}, G_{0}$ and $G_{\mathrm{ext}, 0}$ such that $U_{0}^{s} \equiv U_{0, z}^{f}(x=0, y=0, z)$ verifies

$$
\mathcal{P}\left(U_{0}^{s}\right)+\left(\mathcal{S}_{f_{0}}\right)^{-1}\left(U_{0}^{s}\right)=G_{\mathrm{ext}, 0}
$$

with $\mathcal{N}_{f_{0}}$ defined as in equation (31) and with $\mathcal{S}_{f_{0}}$ defined as $\mathcal{S}_{f_{0}}=\mathcal{T} r \circ \mathcal{N}_{f_{0}}$.

Let us call $U_{n, N}^{s}$ the solution in the finite space $\mathbb{V}_{N}$ of the exact problem (32). Let us define the convergence parameter $\varepsilon_{n, N}=\frac{\left\|U_{n, N}^{s}-U_{0}^{s}\right\|}{\left\|U_{0}^{S}\right\|}$. By construction, we expect $U_{n, N}^{s}$ to converge toward $U_{0}^{s}$ when $n$ or $N$ increases. 


\subsection{2. $3 D$ settings}

In the 3D setting, we consider the polynomial velocity field:

$$
U_{0, x}^{f}=U_{0, y}^{f}=x\left(l_{x}-x\right) y\left(l_{y}-y\right) z\left(l_{z}-z\right), \quad U_{0, z}^{f}=\left(l_{z}-z\right)^{4} x^{2}\left(l_{x}-x\right)^{2} y^{2}\left(l_{y}-y\right)^{2}
$$

For the parameters $n$ and $N$ as low as $n=13$ and $N=2(\mathcal{M}=25$ basis functions), we obtain an error as low as $1.9 \%\left(\varepsilon_{13,2}=0.019\right)$, which is very satisfying considering the low density of nodes in the volume.

\subsection{3. $2 D$ setting}

To limit computational costs, we have only carried out the full convergence study in $2 \mathrm{D}$. We consider the following reference velocity field:

$$
U_{0, x}^{f}=\left(l_{z}-z\right) z\left(l_{x}-x\right)\left(\frac{l_{x}}{2}-x\right) x \quad U_{0, z}^{f}=\left(l_{z}-z\right)^{2} x^{2}\left(l_{x}-x\right)^{2}\left(\frac{l_{x}}{2}-x\right)^{2}
$$

We verify (Figure 3) that $U_{n, N}^{s} \rightarrow U_{0}^{s}$ when $(n, N) \rightarrow(+\infty,+\infty)$. Of course, since $U_{n, N}^{s}$ results from a double approximation process, the accuracy is limited by either one of the approximation errors, which explains the asymptotic behaviour. The error also seems to increase when the number of modes is larger than a maximum value that depends on the mesh size. It is a lock-in phenomenon, which is standard in numerical analysis. 

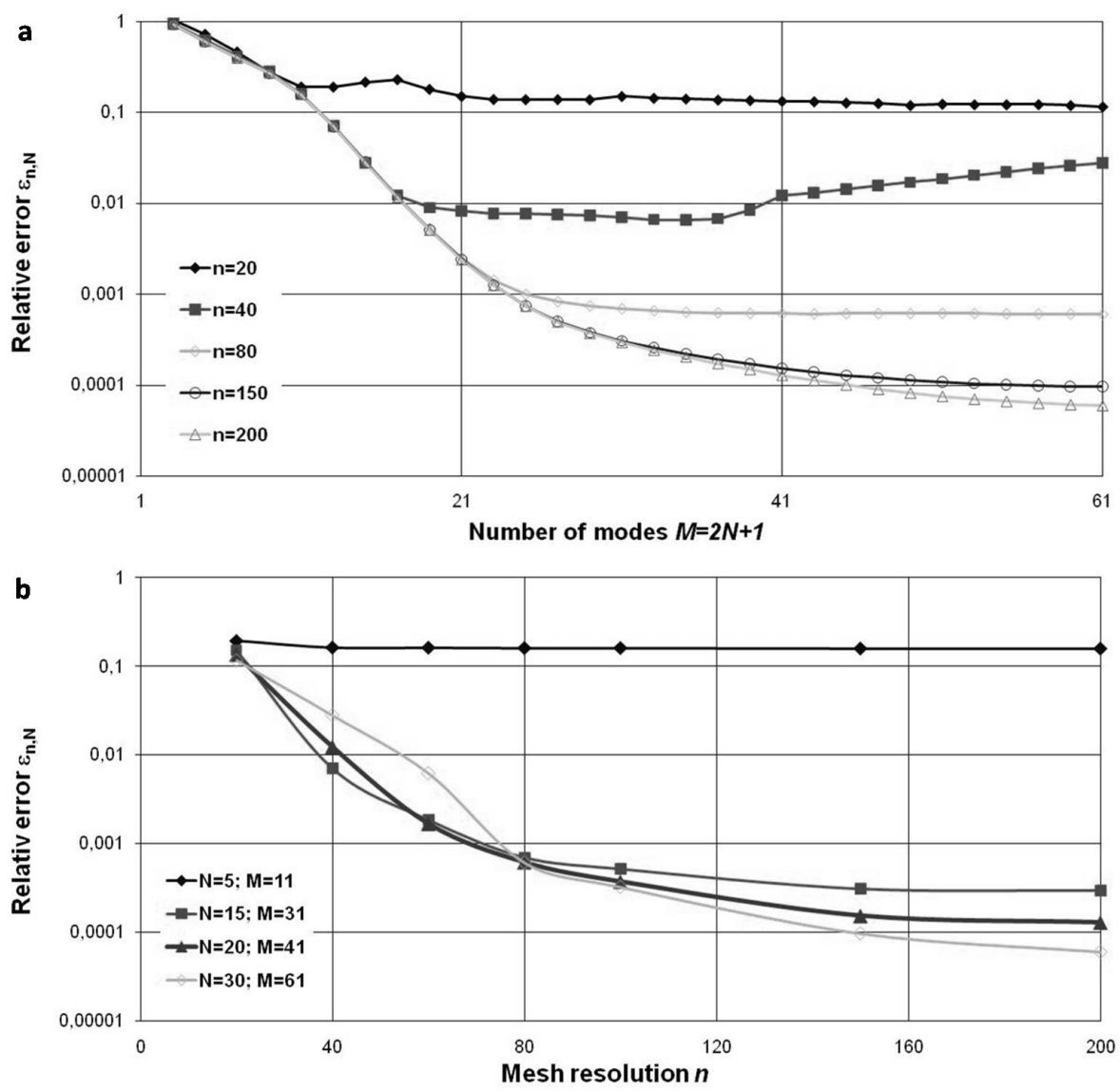

Figure 3: Convergence of the computed 2D solution toward a known polynomial solution: relative error $\varepsilon_{n, N}$ with respect to the number of modes $\mathcal{M}_{2 D}=2 N+1$ for different mesh resolutions $n$ (a) and vice versa (b)

\section{Results}

\subsection{Behaviour of the fluid under harmonic homogeneous load}

In this section, we set $G_{0}=1$ (homogeneous harmonic load on the boundary $\Gamma^{s}$ ) and we compute the pressure and velocity fields $\left(P, \boldsymbol{U}^{f}\right)=\mathcal{N}\left(G_{0}\right)$. Let us define the non-dimensional acoustic admittance $Y(z)$ as the $L_{2}$-norm of the fluid velocity on a section of the domain parallel to $\Gamma^{s}$ (plane $\Gamma_{Z}=\{x, y, z=Z\}$ ) divided by the $L_{2}$-norm of the load, that is $Y(z)=\frac{\sqrt{\int_{\Gamma_{Z}}\left|U_{z}^{f}(x, y, z)\right|^{2}}}{\sqrt{\int_{\Gamma_{Z}}|G|^{2}}}=\sqrt{\int_{\Gamma_{Z}}\left|U_{z}^{f}(x, y, z)\right|^{2}}$

\subsubsection{From a $3 D$ to a $2 D$ setting}

The Figure 4 compares the acoustic admittance $Y(0)\left(\right.$ e.g. the average velocity on $\left.\Gamma^{s}\right)$ of the $3 \mathrm{D}$ and 2D domain presented Figure 1. The 2D and 3D frequency response functions (FRF) display very similar features in terms of amplitudes, resonance frequencies and quality factor of resonances. 


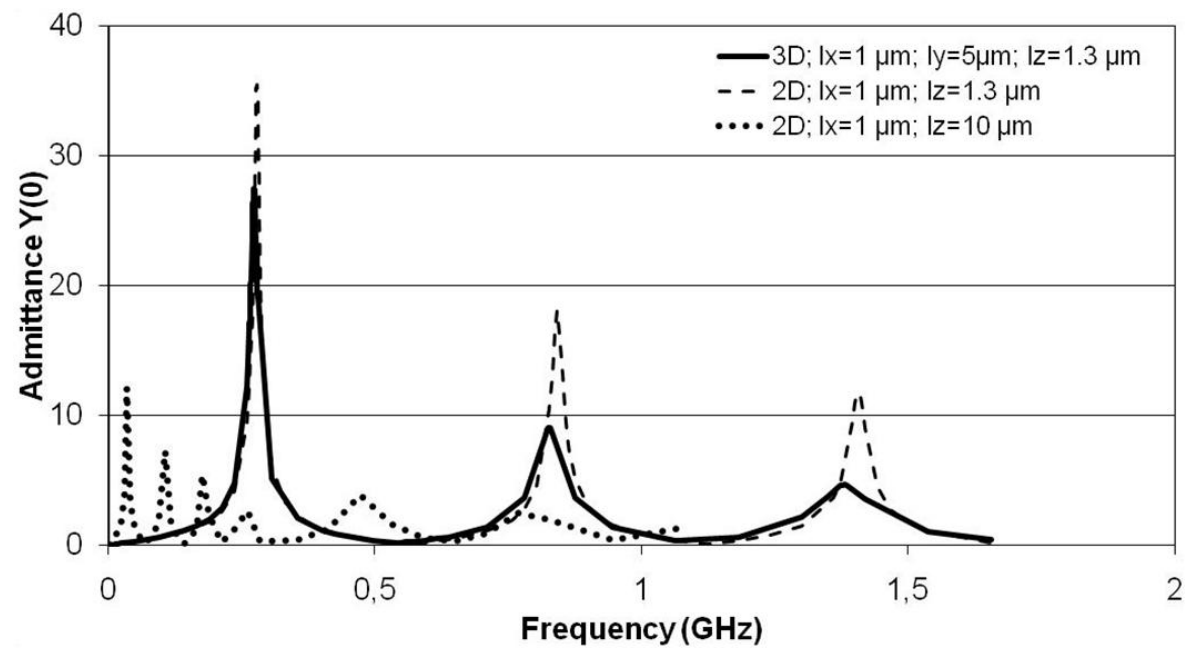

Figure 4: Non-dimensional admittance $Y(0)$ with respect to frequency for a 3D domain of size $l_{x}=1 \mu \mathrm{m}$ $\mathrm{x} l_{y}=1 \mu \mathrm{m} \times l_{z}=1.3 \mu \mathrm{m}$ and two $2 \mathrm{D}$ domains of size $l_{x}=1 \mu \mathrm{m} \times l_{z}=1.3 \mu \mathrm{m}$ and $l_{x}=1 \mu \mathrm{m} \times l_{z}=10 \mu \mathrm{m}$.

Quantitatively, the Figure 5 shows that, as expected, the 3D resonance frequencies and quality factors shift toward the 2D limit values when the depth $l_{y}$ increases (system translation-invariant along the y-axis). The $3 \mathrm{D}$ velocity profiles $U_{z}^{f, 3 D}\left(x, \frac{l_{y}}{2}, z\right)$ appear to converge toward the $2 \mathrm{D}$ velocity profile $U_{z}^{f, 2 D}(x, z)$ when $l_{y}$ increases. For instance, at $210 \mathrm{MHz}$, the (norm 2) relative error between $U_{z}^{f, 3 D}\left(x, \frac{l_{y}}{2}, 0\right)$ and $U_{z}^{f, 2 D}(x, 0)$ decreases by a factor 2 between $l_{y}=1 \mu \mathrm{m}$ and $l_{y}=5 \mu \mathrm{m}$, going from $16 \%$ down to $8 \%$.

Considering the qualitative agreement between 2D and 3D frequency responses, we will work only in a $2 \mathrm{D}$ setting in the rest of this study.
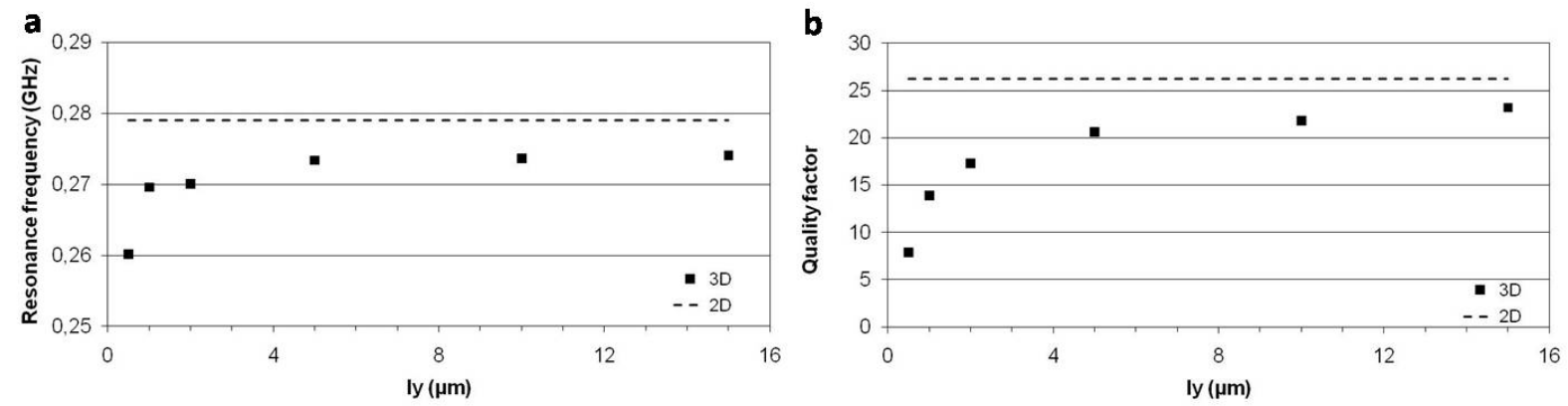

Figure 5: Frequency (a) and quality factor (b) of the first resonance for 3D domains of same height $l_{x}=1 \mu \mathrm{m}$, same width $l_{z}=1.3 \mu \mathrm{m}$ and different depth $l_{y}$ (solid lines). As expected, when $l_{y}$ increases, the $3 \mathrm{D}$ values shift toward the $2 \mathrm{D}$ limit values (dashed line) 


\subsubsection{Visco-acoustic resonances}

The Figure 6 shows the velocity profiles $\left|U_{x}^{f}\right|$ and $\left|U_{z}^{f}\right|$ of the first three modes identified on the FRF of the $l_{z}=1.3 \mu \mathrm{m}$ domain displayed on the Figure 4 . The velocity profiles $\left|U_{x}^{f}\right|$ and $\left|U_{z}^{f}\right|$ of the $i$-th mode have respectively $i$ and $i-1$ nodes, just like in non-viscous acoustics.

In non-viscous acoustics, the water-filled domain of width $l_{z}$ would resonate at the frequencies $f_{k}=\frac{c}{\lambda_{k}}$, with $\lambda_{k}=\frac{4 l_{z}}{1+2 k}, \quad k \in \mathbb{N}$. A quantitative analysis of the FRF presented Figure 4 indicates that the frequencies of the visco-acoustic resonances are close to the frequencies $f_{k}$ of the non-viscous resonances. For instance, for $l_{z}=1.3 \mu \mathrm{m}$, the first resonance is down-shifted by only $3.1 \% \pm 0.5 \%$ (Figure 7). The quality factor of the first resonance is $17.2 \pm 14.3 \%$.

Table 1 sums up the resonance shifts and the resonance quality factors computed for different fluid parameters. It shows that the frequency downshift increases and the quality factor decreases with decreasing parameter Re, i.e when the role of the viscous terms in Navier-Stokes equations increases. Note that the errors given in Table 1 result from the various non-dimensional frequency steps $\delta \omega$ used for the computation of $Y_{0}$ with respect to $\omega$.

\begin{tabular}{|c|c|c|c|c|c|c|c|}
\hline$\frac{L}{L_{0}}$ & $\frac{\rho^{f}}{\rho_{0}^{f}}$ & $\frac{p}{p_{0}}$ & $\frac{\kappa_{T}}{\kappa_{T, 0}}$ & $\frac{\mu}{\mu_{0}}$ & $\frac{R e}{R e_{0}}$ & Frequency downshift (\%) & Quality factor \\
\hline 5 & 1 & 1 & 1 & 1 & 5 & $1.6 \pm 0.2$ & $150 \pm 18 \%$ \\
1 & 10 & 1 & 1 & 1 & $\sqrt{(10)}$ & $2.7 \pm 0.5$ & $29 \pm 18 \%$ \\
1 & 1 & 1 & 1 & 1 & 1 & $3.1 \pm 0.5$ & $17 \pm 14 \%$ \\
1 & 1 & 10 & 1 & 1 & 1 & $3.1 \pm 0.5$ & $17 \pm 14 \%$ \\
1 & 1 & 1 & 10 & 1 & $\frac{1}{\sqrt{10}}$ & $4.4 \pm 0.5$ & $8.4 \pm 7 \%$ \\
1 & 1 & 1 & 1 & 10 & $\frac{1}{10}$ & $6.4 \pm 1.0$ & $4.4 \pm 8 \%$ \\
\hline
\end{tabular}

Table 1: Frequency downshift and quality factor of first resonance with respect to various parameters. Here, the value of the parameter $X_{0}$ is the reference value of the parameter $X$ as given in paragraph 3.2.3.

\subsubsection{Boundary layer}

We identify (Figure 8) two types of velocity profiles along the $x$-axis depending on the excitation frequency. At frequencies $f$ much lower than the first resonance $\left(f<<\frac{c}{4 l_{z}}\right)$, viscous effects dominate over inertial effects. The velocity profile $\left|U_{z}^{f}(x, z=k)\right|$ along the $x$-direction is parabolic. Beyond a 

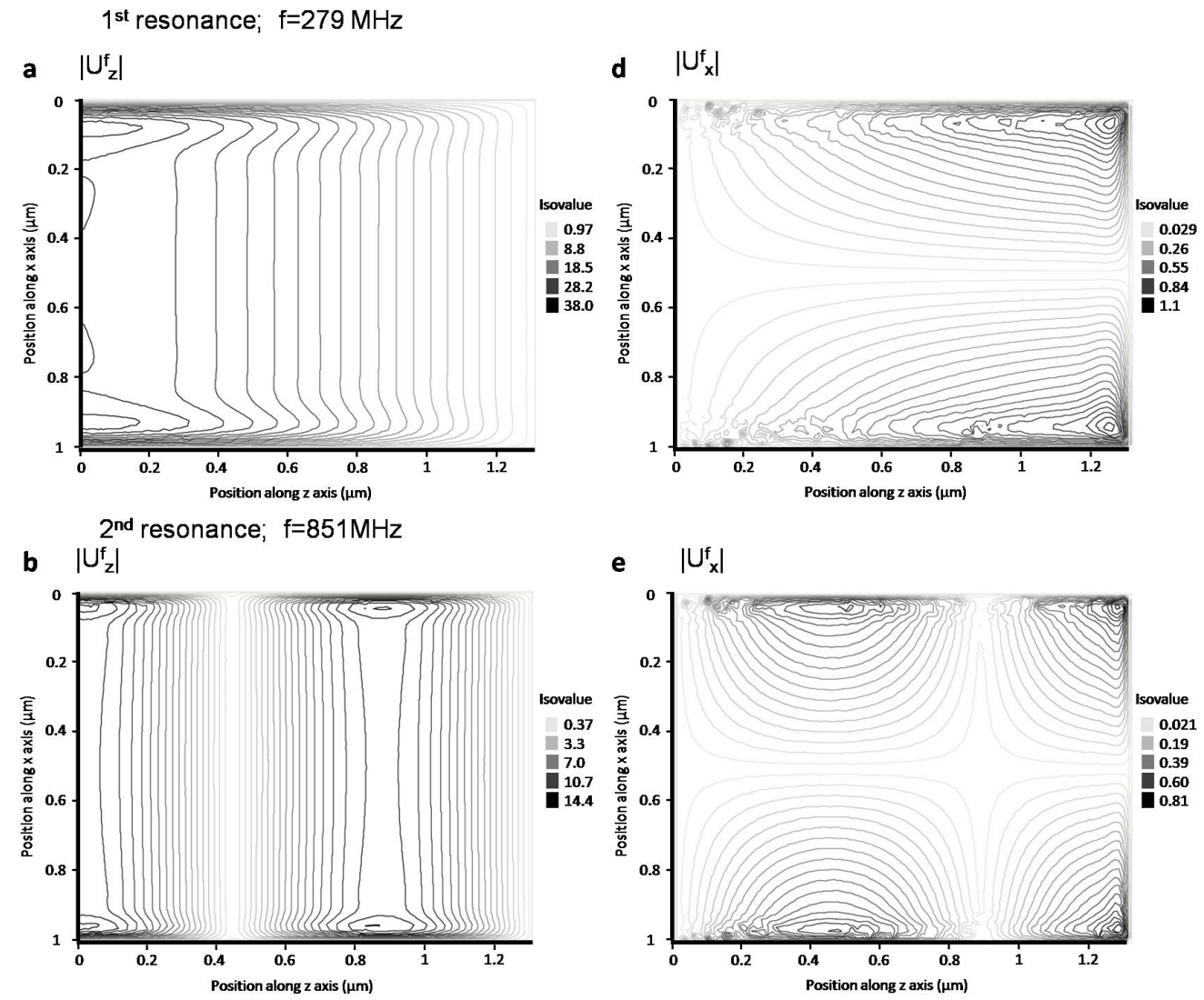

e $\quad\left|U_{x}^{f}\right|$

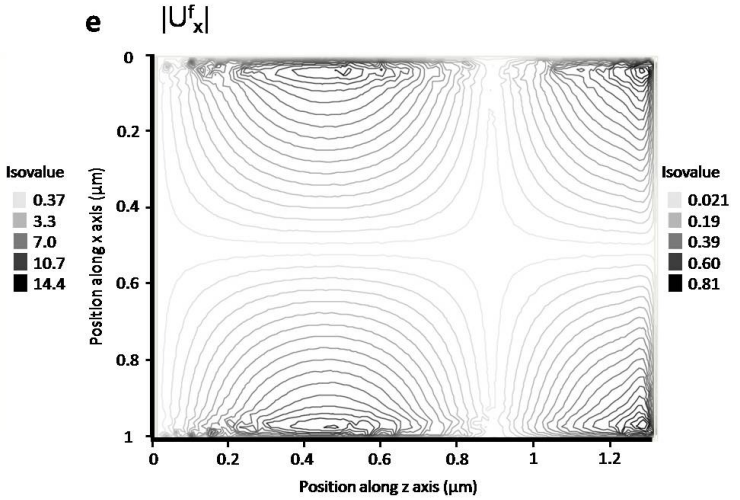

c $\quad\left|\mathrm{U}_{\mathrm{z}}^{\mathrm{f}}\right|$
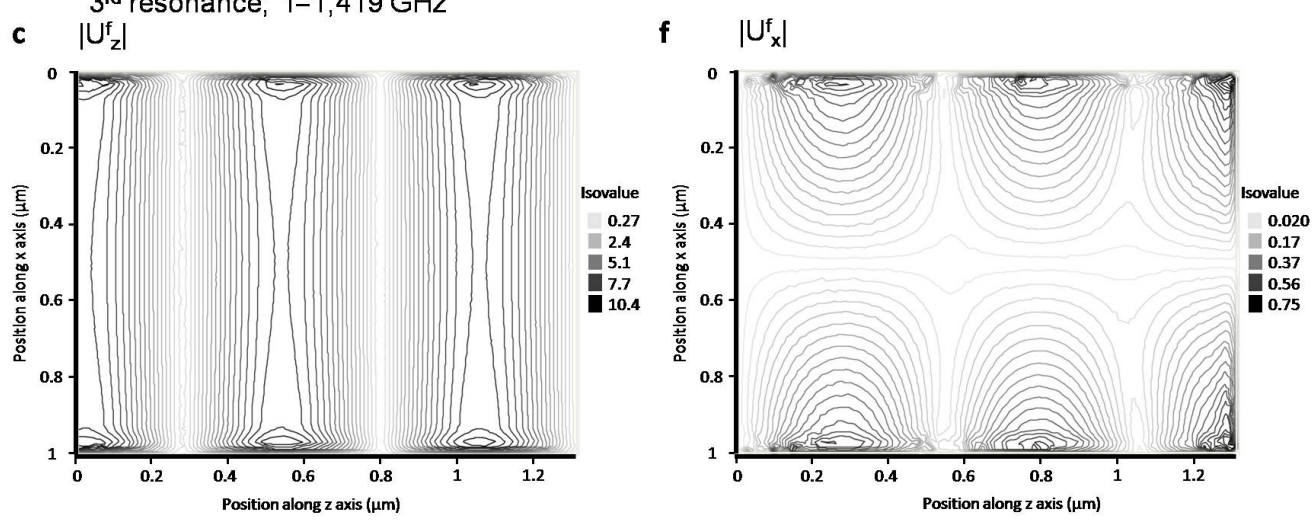

Figure 6: Isovalue lines of the non-dimensional velocity fields $\left|U_{z}^{f}\right|$ (a-c) and $\left|U_{x}^{f}\right|$ (d-f) at the first three resonances, for a domain of width $1 \mu \mathrm{m}$ and length $1.3 \mu \mathrm{m}$. 


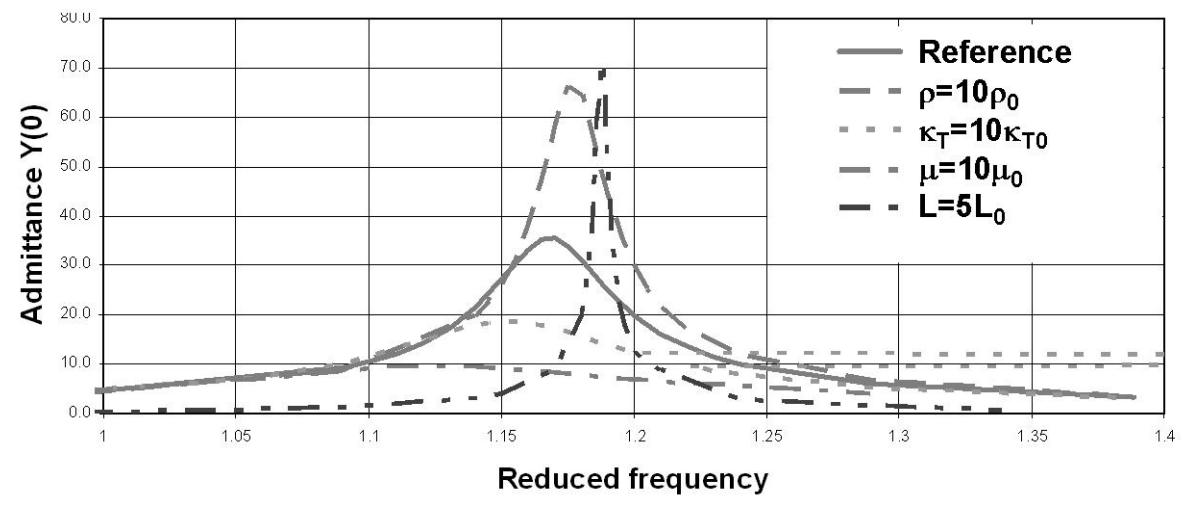

Figure 7: Non-dimensional admittance $Y(0)$ at frequencies close to first resonance for various parameters. The domain length is $1.3 \mu \mathrm{m}$.

limit frequency $f_{\text {lim }}$, inertial effects dominate over viscous effects. The velocity $\left|U_{z}^{f}(x, z=k)\right|$ is constant outside the boundary layer, that is delimited by one velocity peak on each side of the domain. For a $1 \mu \mathrm{m} \times 1.3 \mu \mathrm{m}$ rectangular domain, the frequency transition occurs between $15 \mathrm{et} 20 \mathrm{MHz}$, at about $5 \%$ of the frequency of the first resonance $f_{1}=\frac{c}{4 l_{z}}$.

The existence of both types of profiles has been pointed out by Beltman in [12] using the low reducedfrequency model. However in water at room temperature and ambient pressure this model is restricted to frequencies much lower than $250 \mathrm{MHz}$. Our calculations up to $2 \mathrm{GHz}$ using the full linearized NavierStokes equations seem to indicate that the behaviour identified at lower frequencies is actually valid over a much larger range of frequencies. 


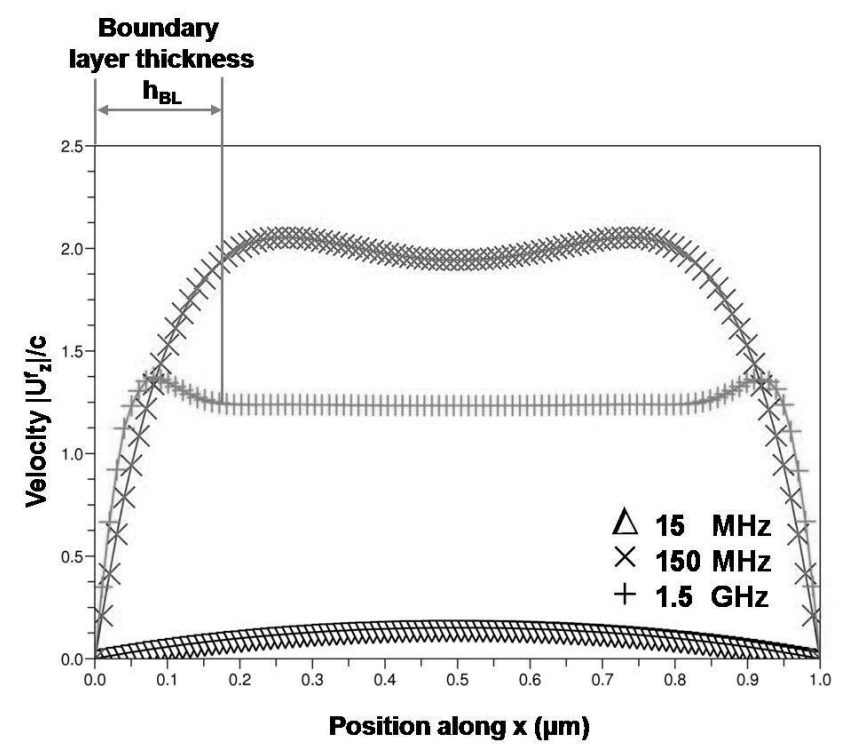

Figure 8: Non-dimensional velocity profile at $z=0$ for different frequencies for a domain of height $l_{x}=1$ $\mu \mathrm{m}$ and width $l_{z}=1.3 \mu \mathrm{m}$

Let us define the (dimensional) boundary layer thickness $h_{B L}$ as

$$
\begin{array}{r}
h_{\mathrm{BL}}=\frac{1}{2}\left(l_{x}-\int_{0}^{l_{x}} \chi(x)\right) \\
\text { and } \quad \mathbb{D}=\left\{x \in\left[0, l_{x}\right] \quad \text { such that } \quad 0.95\left|U_{z}^{f}\left(\frac{l_{x}}{2}, 0\right)\right| \leq\left|U_{z}^{f}(x, 0)\right| \leq 1.05\left|U_{z}^{f}\left(\frac{l_{x}}{2}, 0\right)\right|\right\}
\end{array}
$$

The domain $\mathbb{D}$ defines the interval along the $x$-axis along which the velocity is constant with $5 \%$ accuracy, and $\chi$ is the characteristic function for the $1 \mathrm{D}$ domain $\mathbb{D}$. The quantity $h_{\mathrm{BL}}$ is thus defined as the thickness of the layer along the boundary where the velocity is not constant.

A large value for $h_{\mathrm{BL}}$ is characteristic of strong viscous effects. The Figure 10 displays $h_{\mathrm{BL}}$ with respect to the frequency $f$. The plot presents definite peaks, approximately localized at the wavelengths $\lambda_{\kappa} \approx \frac{2 l_{z}}{\kappa}, \quad \kappa \in \mathbb{N}$, that is to say halfway between successive resonance frequencies. At these 'antiresonancies', the viscosity effect is maximal and the admittance is minimal. Between successive 'antiresonancies', $h_{\mathrm{BL}}$ decreases with increasing frequency and becomes stationary around $\lambda_{k} \approx \frac{4 l_{z}}{2 k+1}$ : close to the resonance, the inertia terms play in the solution a dominant role over the viscosity terms. 


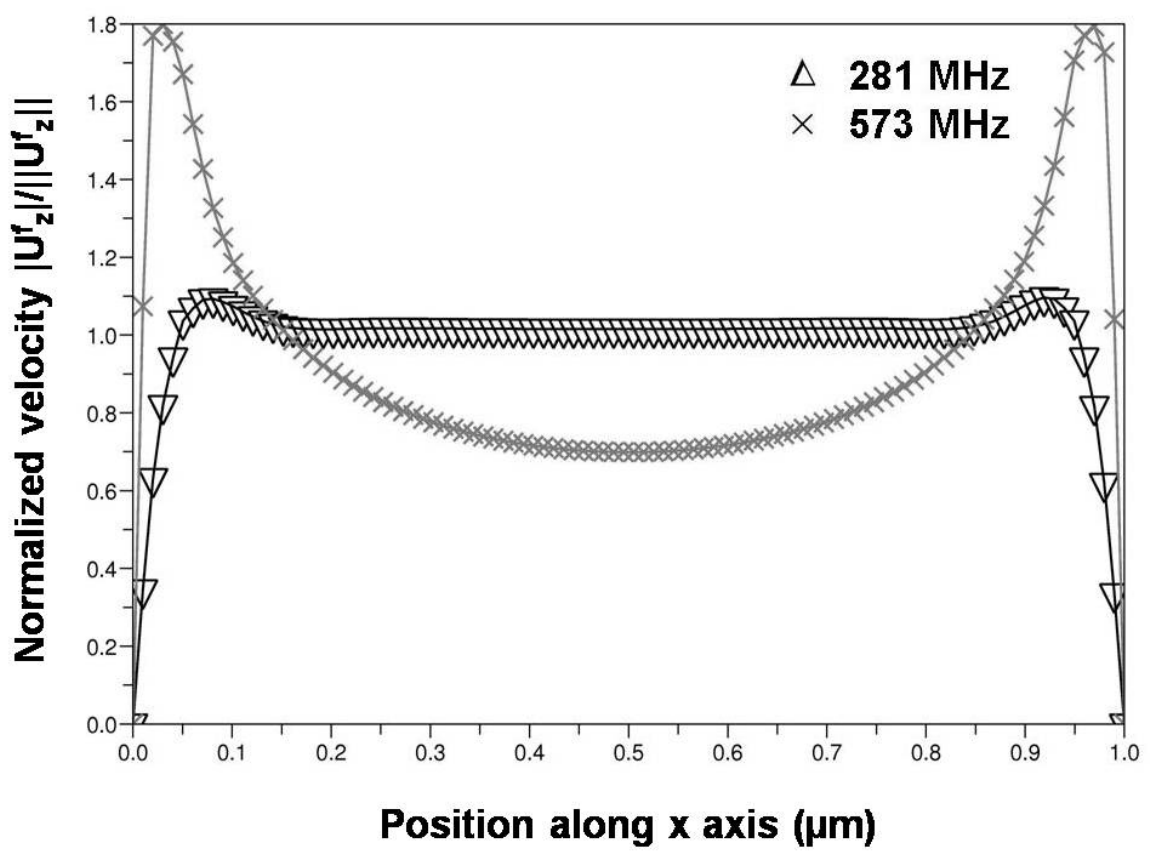

Figure 9: Normalized velocity profile at $z=0$ at first resonance $(281 \mathrm{MHz})$ and at first "antiresonance" (573 $\mathrm{MHz}$ ) for a domain of height $l_{x}=1 \mu \mathrm{m}$ and width $l_{z}=1.3 \mu \mathrm{m}$

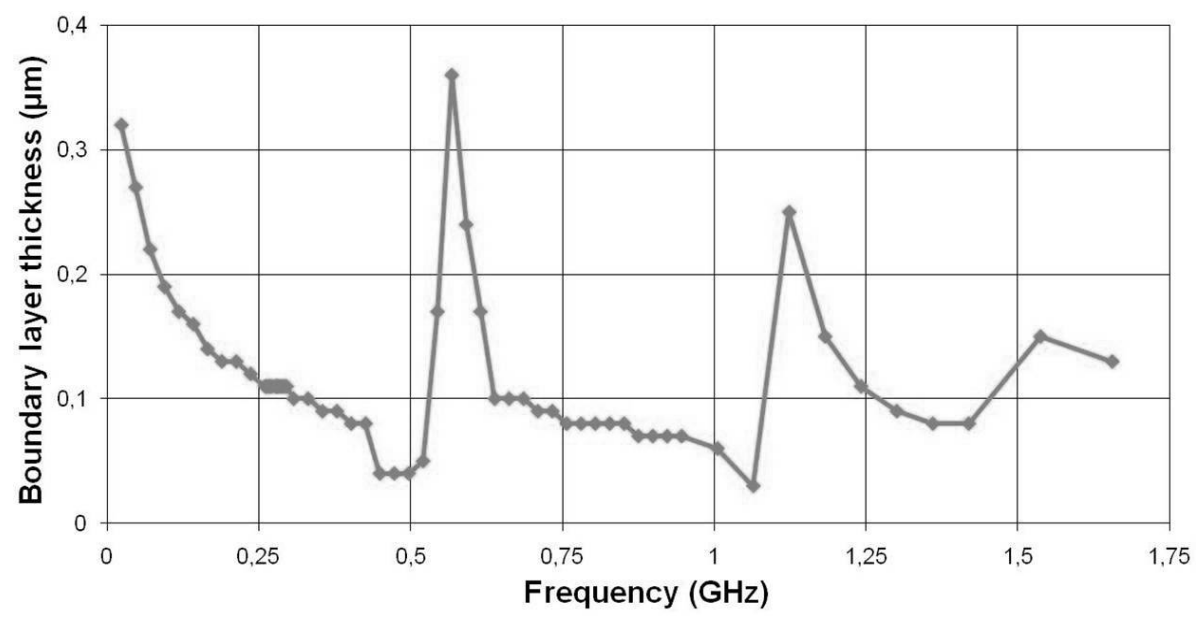

Figure 10: Boundary layer thickness $h_{B L}$ with respect to frequency for a domain of height $l_{x}=1 \mu \mathrm{m}$ and width $l_{z}=1.3 \mu \mathrm{m}$

\subsection{Behaviour of the elasto-acoustic system under harmonic homogeneous load}

We consider in this section the fluid-plate system under an harmonic homogeneous load of amplitude $G_{0}=-8000 \mathrm{~Pa}$. We study the plate displacement $D^{s}=\frac{1}{q} U^{s} \in H_{0}^{2}\left(\Gamma^{s}\right)$ solution of equation (22). The results are analyzed by computing the $L^{2}$-norm of the plate displacement $D^{s}$, denoted $\left\|D^{s}\right\|=\left(\int_{0}^{1}\left|D^{s}(y)\right|^{2}\right)^{\frac{1}{2}}$. 


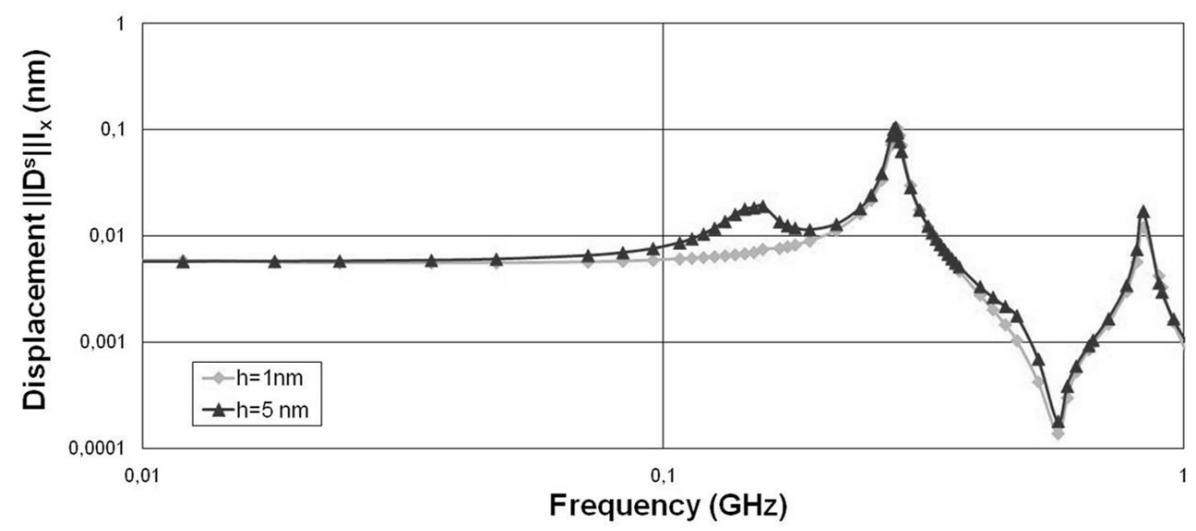

Figure 11: Norm of the dimensional plate displacements $\left\|D^{s}\right\| l_{x}$ with respect to frequency for a domain of width $l_{x}=1 \mu \mathrm{m}$ and length $l_{z}=1.3 \mu \mathrm{m}$

\subsubsection{Fluid resonances}

All the resonances identified in the fluid-only system also appear in the fluid-plate system FRF, which is, as a consequence, very sensitive to pore width. The frequency shift between the first three fluid-structure and fluid-only resonances is lower than $2 \%$; the quality factors of the fluid-structure resonances are lower than in the fluid-only system $(Q=12 \pm 33 \%$ for the first resonance). The frequency and quality factor of these resonances are not sensitive to the plate characteristics (Young's modulus, Poisson's ratio, density, thickness).

The shape of these fluide-plate modes (Figure 12) is similar to the shape of the fluid-only modes (Figure 8). For instance, the Figure 12 displays the profile of the plate vibrations $\left|D^{s}\right|$ at the first resonance computed from 23 basis functions. The lateral velocity peaks are apparent, as well as an approximately 'flat' portion between the peaks. The oscillations in this 'flat' portion of the profile are due to the Fourier approximation.

\subsubsection{Plate resonances and added stiffness effect}

These elements indicate that the fluid resonances are only weakly perturbed by the presence of the plate. On the contrary, the plate resonances are strongly perturbed by the presence of the fluid. Indeed, for a very thin plate $(h<5 \mathrm{~nm})$, no resonance can be observed in the FRF. When the plate thickness $h$ increases $(h=5 \mathrm{~nm})$, one of the resonances of the FRF appears at a frequency significantly different from the frequencies of the fluid resonances (Figure 11). The frequency and amplitude of this resonance strongly depend on the parameters of the plate $E, \rho^{s}, h$ and $l_{x}$. 


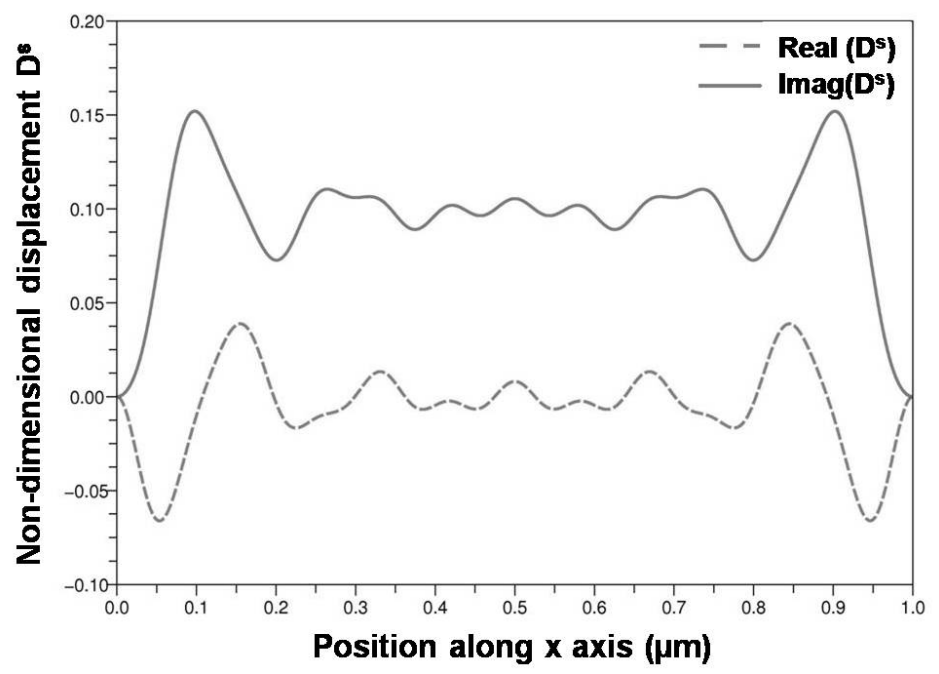

Figure 12: Displacement profile $\left|D^{s}\right|$ at $281 \mathrm{MHz}$ (first resonance), computed for $\mathrm{N}=11$ (23 modes) and $n=100$.

According to the Figure 13 presenting $\left|D^{s}(x)\right|$ with respect to $x$ at $155 \mathrm{MHz}$ for a plate of thickness $5 \mathrm{~nm}$, Young's modulus $1 \mathrm{TPa}$ and density 2 , the observed resonance could either be the first or the third resonance of the plate (the second is excluded for symmetry reasons). In vacuum, the resonance frequencies of a plate are given by the formula $\phi_{i}^{2}=\alpha_{i} \frac{E h^{2}}{\left(1-v^{2}\right) \rho^{s} L^{4}}+\frac{g_{1}}{a_{p}}$ with $\alpha_{1}=1,027, \alpha_{2}=2,756$ and $\alpha_{3}=5,404$ [39]. It leads to $\phi_{1}=121 \mathrm{MHz}, \phi_{2}=331 \mathrm{MHz}$ and $\phi_{3}=648 \mathrm{MHz}$ in our case.

If the observed resonance was the third plate resonance, its frequency downshift ratio would be $75 \%$. Assuming that a similar downshift ratio could be applied to the first resonance, we should observe this first resonance around $30 \mathrm{MHz}$, which is not the case. This means that the structure mode we observe is the first plate mode, strongly perturbed and upshifted by $13 \%$ : the water load causes an added stiffness effect.

Compared to the situation in air described in [20], it appears that the plate modes are much more strongly perturbed in water than in air, which was to be expected. There is however a similar added stiffness effect. 


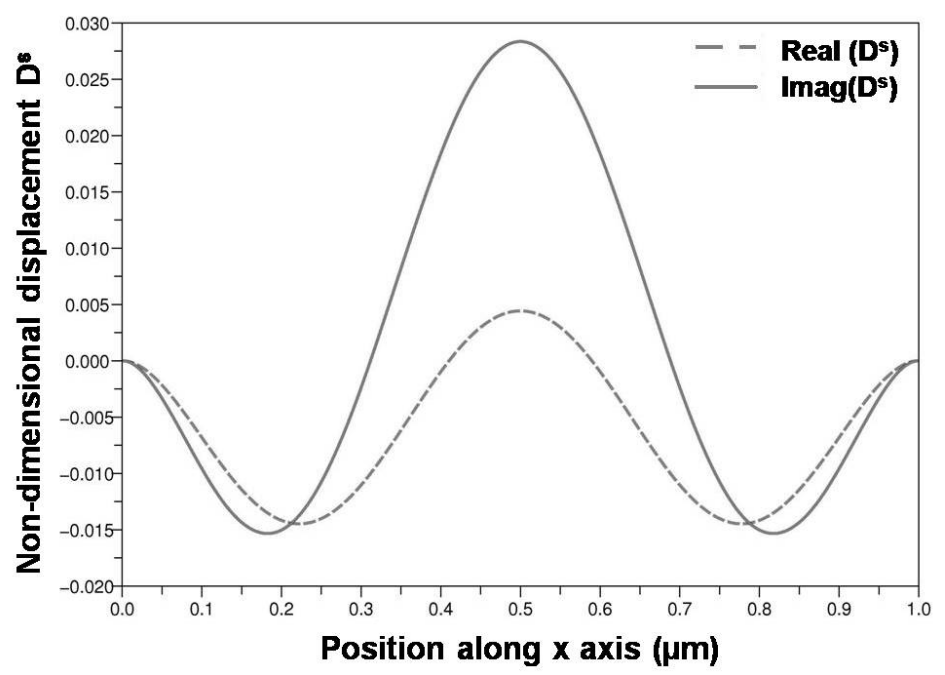

Figure 13: Displacement profile $\left|D^{s}\right|$ of a $h=5 \mathrm{~nm}$ thick plate at $155 \mathrm{MHz}$, frequency associated to a plate resonance

\section{Conclusions}

We have developed a computational method for the modelling of a microplate vibrating between 10 $\mathrm{MHz}$ and $2 \mathrm{GHz}$ in a water-filled domain of micrometric size. The method is based on the condensation of the fluid part of the fluid-structure equations on the structure by a finite element method, and on a spectral approximation of the structural equations. We have proved the well-posedness of the problem, numerically studied its convergence and analyzed the results provided by the method.

Although viscous terms occasion a frequency downshift and a decrease of the quality factors, the fluid domain is still resonant at frequencies close to the resonance frequencies observed in a non viscous acoustic cavity. The resonances observed for the system coupled with a thin plate are at the same frequency as the resonances of the fluid alone. When the plate thickness increases, the fluid domain resonances do not shift. However, a low quality factor resonance appears. It seems to be the strongly upshifted fundamental mode of the plate. Altogether, the frequency response function of the system is strongly sensitive to the pore width, which makes the device a good candidate for porosity monitoring.

In a further study, geometries will have to be complexified to account for more realistic pore shape in cementitious materials. Eventually, results will have to be interpreted in terms of observability of the characteristics of the porosity for cementitious materials instrumentation. 


\section{References}

[1] P. C. Chang, A. Flatau, S. Liu, Health monitoring in civil infrastructure, Structural health monitoring 2 (3) (2003) 257-267.

[2] D. M. McCann, M. C. Forde, Review of ndt methods in the assessment of concrete and masonry structures, NDT\&E International 34 (2001) 71-84.

[3] B. Lebental, E. Delevoye, A.Ghis, Procédé et dispositif d'analyse acoustique de microporosites dans un matériau tel que le béton à l'aide d'une pluralité de transducteurs cmuts incorporés dans le matériau (process and device for acoustical analysis of microporosities in a material such as concrete using embedded cmut transducers), patent submitted n en 0857927 (2008).

[4] B. Lebental, F. Bourquin, J.-M. Caussignac, L. Acauan, S. Sanaur, A. Ghis, In-situ non destructive testing of cementitous materials via embedded ultrasonic transducers made up of carbon nanotubes., in: NDTCE'09, Non-Destructive Testing in Civil Engineering, 2009.

[5] B. Lebental, A. Ghis, Cellule cmut formée d'une membrane de nanotubes ou de nanofils ou de nanopoutres et dispositif d'imagerie acoustique ultra haute fréquence comprenant une pluralité de telles cellules (cmut cell formed by a nanotubes or nanowires or nanobeams membrane, and ultra high frequency acoustic imaging device incorporating several of these cells), patent submitted $\mathrm{n}^{\circ}$ en 0857928 (2008).

[6] R. Hilfer, Transport and relaxation phenomena in porous media, Advances in Chemical Physics 92 (1995) 1-225.

[7] S. Xu, X. Yue, J. R. Hou, Experimental investigation on flow characteristics of deionized water in microtubes, Chinese science bulletin 52 (6) (2007) 849-854.

[8] I. Papautsky, T. Ameel, A. B. Frazier, A review of laminar single-phase flow in microchannels, in: Proceedings of 2001 ASME International Mechanical Engineering Congress and Exposition, New-York, USA, 2001.

[9] H. Wijshoff, Structure- and fluid-dynamics in piezo inkjet printheads, Ph.D. thesis, University of Twente, Ensschede, The Netherlands (2008). 
[10] L. D. Landau, E. M. Lifshitz, Fluid Mechanics; 2nd Edition, Pergamon Press, 1959.

[11] M. Bruneau, P. Herzog, J. Kergomard, J. D. Polack, General formulation of the dispersion equation in bounded visco-thermal fluid, and application to some simple geometries, Wave motion 11 (1989) $441-451$.

[12] W. M. Beltman, Viscothermal wave propagation including acousto-elastic interaction, part i: theory, Journal of Sound and Vibration 227 (3) (1999) 555-586.

[13] W. M. Beltman, Viscothermal wave propagation including acousto-elastic interaction, part ii: application, Journal of Sound and Vibration 227 (3) (1999) 587-609.

[14] M. Malinen, M. Lyly, P. Raback, A. Käarkkäinen, L. Käarkkäinen, A finite element method for the modeling of thermo-viscous effects in acoustics, in: European Congress on Computational Methods in Applied Sciences and Engineering ECCOMAS 2004, Jyväskylä, Finland, 24-28 July, 2004.

[15] W. R. Kampinga, Y. H. Wijnant, A. de Boer, Performance of several viscothermal acoustic finite elements, Acta Acustica united with Acustica 96 (2010) 115-124.

[16] R. Kampinga, Viscothermal acoustics using finite elements. analysis tools for engineers, Ph.D. thesis, University of Twente, Ensschede, The Netherlands (2010).

[17] M. J. J. Nijhof, Y. H. Wijnant, A. de Boer, An acoustic finite element including viscothermal effects, in: 14th International Congress on Sound and Vibration, Cairns, Australia, 9-12 July, 2007.

[18] C. Karra, M. B. Tahar, An integral equation formulation for boundary element analysis of propagation in viscothermal fluids, Journal Acoustical Society of America 102 (1997) 1311-1318.

[19] R. Christensen, P. Juhl, V. C. Henriquez, Practical modeling of acoustic losses in air due to heat conduction and viscosity, in: Acoustics'08, June 29-July 4, Paris, France, 2008.

[20] W. M. Beltman, P. J. M. V. D. Hoogt, R. M. E. J. Spierinfg, H. Tijdeman, Implementation and experimental validation of a new viscothermal acoustic finite element for acousto-elastic problems, Journal of Sound and Vibration 216 (1) (1998) 159-185. 
[21] C. Karra, M. B. Tahar, Boundary element analysis of vibro-acoustic interaction between vibrating membrane and thin fluid layer, Flow, Turbulence and Combustion 61 (1999) 179-187.

[22] R. W. Barber, D. R. Emerson, The influence of knudsen number on the hydrodynamic development length within parallel plate micro-channels, Advances in Fluid Mechanics 4 (2002) 207-216.

[23] W. Qu, G. M. Mala, L. Dongqing, Pressure-driven water flows in trapezoidal silicon microchannels, International Journal of Heat and Mass Transfer 43 (2000) 353-364.

[24] X.-B. Mi, A. T. Chwang, Molecular dynamics simulations of nanochannel flows at low reynolds numbers, Molecules 8 (2003) 193-206.

[25] X. B. Nie, S. Y. Chen, W. N. E, M. O. Robbins, A continuum and molecular dynamics hybrid method for micro- and nano-fluid flow, Journal of Fluid Mechanics 500 (2004) 55-64.

[26] H. Daiguji, Ion transport in nanofluidic channels, Chemical Society Reviews 39 (2010) 901-911.

[27] J. Gaudioso, H. G. Craighead, Characterizing electroosmotic flow in microfluidic devices, Journal of Chromatography 971 (2002) 249-253.

[28] B. J. Kirby, E. F. H. Jr., Zeta potential of microfluidic substrates: 1. theory, experimental techniques, and effects on separations, Electrophoresis 25 (2004) 187-202.

[29] W. Sparreboom, A. V. D. Berg, J. C. T. Eijkel, Principles and applications of nanofluidic transport, Nature Nanotechnology 4 (2009) 713-720.

[30] J. Koo, C. Kleinstreuer, Liquid flow in microchannels: experimental observations and computational analyses of microfluidics effects, Journal of Micromechanics and Microengineering 13 (2003) 568-579.

[31] R. Dautray, J.-L. Lions, Mathematical analysis and numerical methods for science and technology, Vol. 5; Evolution Problems 1, Springer Verlag, 2000.

[32] A. Krishnan, E.Dujardin, T. W. Ebbesen, P. N. Yianilos, M. M. J. Treacy, Young's modulus of single-walled nanotubes, Physical Review B 58 (1998) 14013.

[33] L. D. Landau, E. M. Lifshitz, Electrodynamics of continuous media, Pergamon Press, 1960. 
[34] F. Hecht, O. Pironneau, A. L. Hyaric, K. Ohtsuka, FreeFem++, 2nd edition, Version 2.24, Laboratoire Jacques-Louis Lions, UPMC, Paris.

[35] S. Hang, TetGen Users' Guide: A quality Tetrahedral Mesh Generator and Three-Dimensional Delaunay Triangulator.

URL http://tetgen.berlios.de

[36] D. N. Arnold, F. Brezzi, Mixed and nonconforming finite element methods implementation, postprocessing and error estimates, Mathematical Modelling and Numerical Analysis 19 (1) (1985) $7-32$.

[37] T. A. Davis, UMFPACK User Guide, Dept. of Computer and Information Science and Engineering Univ. of Florida, Gainesville, FL.

URL http://www.cise.ufl.edu/research/sparse/umfpack/

[38] E. Anderson, Z. Ba, C. Bischof, S. Blackford, J. Demmel, J. Dongarra, J. D. Croz, A. Greenbaum, S. Hammarling, A. McKenney, D. Sorensen, LAPACK Users' Guide Third Edition, Society for Industrial and Applied Mathematics.

URL http://www.netlib.org/lapack/lug/

[39] A. N. Cleland, M. L. Roukes, Noise processes in nanomechanical resonators, Journal of Applied Physics 92 (5) (2002) 2758-2769. 


\section{List of Figures}

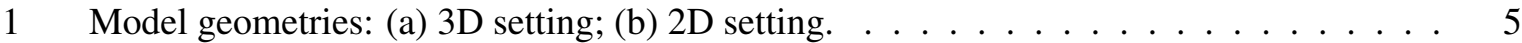

2 Convergence of the computed solution toward a known polynomial solution: relative errors $\varepsilon_{x}$ and $\varepsilon_{z}$ with respect to the mesh resolution $n \ldots \ldots \ldots$

3 Convergence of the computed 2D solution toward a known polynomial solution: relative error $\varepsilon_{n, N}$ with respect to the number of modes $\mathcal{M}_{2 D}=2 N+1$ for different mesh resolutions $n$ (a) and vice versa $(\mathrm{b}) \ldots \ldots \ldots \ldots \ldots$

4 Non-dimensional admittance $Y(0)$ with respect to frequency for a 3D domain of size $l_{x}=1$ $\mu \mathrm{m} \times l_{y}=1 \mu \mathrm{m} \times l_{z}=1.3 \mu \mathrm{m}$ and two $2 \mathrm{D}$ domains of size $l_{x}=1 \mu \mathrm{m} \times l_{z}=1.3 \mu \mathrm{m}$ and $l_{x}=1 \mu \mathrm{m}$

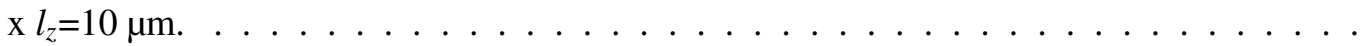

5 Frequency (a) and quality factor (b) of the first resonance for 3D domains of same height $l_{x}=1 \mu \mathrm{m}$, same width $l_{z}=1.3 \mu \mathrm{m}$ and different depth $l_{y}$ (solid lines). As expected, when $l_{y}$ increases, the $3 \mathrm{D}$ values shift toward the $2 \mathrm{D}$ limit values (dashed line) $\ldots \ldots \ldots$

6 Isovalue lines of the non-dimensional velocity fields $\left|U_{z}^{f}\right|$ (a-c) and $\left|U_{x}^{f}\right|$ (d-f) at the first three resonances, for a domain of width $1 \mu \mathrm{m}$ and length $1.3 \mu \mathrm{m}$.

7 Non-dimensional admittance $Y(0)$ at frequencies close to first resonance for various parameters. The domain length is $1.3 \mu \mathrm{m} . \ldots \ldots \ldots \ldots \ldots$

8 Non-dimensional velocity profile at $z=0$ for different frequencies for a domain of height $l_{x}=1 \mu \mathrm{m}$ and width $l_{z}=1.3 \mu \mathrm{m} \ldots \ldots \ldots \ldots \ldots$

9 Normalized velocity profile at $z=0$ at first resonance $(281 \mathrm{MHz})$ and at first "antiresonance" $(573 \mathrm{MHz})$ for a domain of height $l_{x}=1 \mu \mathrm{m}$ and width $l_{z}=1.3 \mu \mathrm{m} \ldots \ldots$. .

10 Boundary layer thickness $h_{B L}$ with respect to frequency for a domain of height $l_{x}=1 \mu \mathrm{m}$

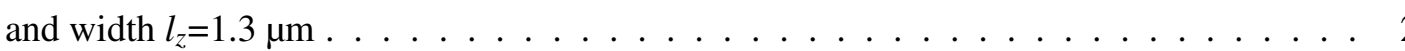

11 Norm of the dimensional plate displacements $\left\|D^{s}\right\| l_{x}$ with respect to frequency for a domain of width $l_{x}=1 \mu \mathrm{m}$ and length $l_{z}=1.3 \mu \mathrm{m} \ldots \ldots \ldots$

12 Displacement profile $\left|D^{s}\right|$ at $281 \mathrm{MHz}$ (first resonance), computed for $\mathrm{N}=11$ (23 modes) and $\mathrm{n}=100$.

13 Displacement profile $\left|D^{s}\right|$ of a $h=5 \mathrm{~nm}$ thick plate at $155 \mathrm{MHz}$, frequency associated to

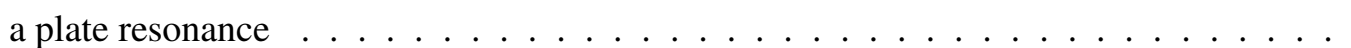




\section{List of Tables}

1 Frequency downshift and quality factor of first resonance with respect to various parameters. Here, the value of the parameter $X_{0}$ is the reference value of the parameter $X$ as

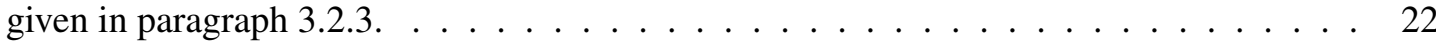

